\title{
Interplay of chemical disorder and electronic inhomogeneity in unconventional superconductors
}

\section{Citation}

Zeljkovic, Ilija, and Jennifer E. Hoffman. 2013. “Interplay of Chemical Disorder and Electronic Inhomogeneity in Unconventional Superconductors." Physical Chemistry Chemical Physics 15 (32): 13462.

\section{Published Version}

doi:10.1039/c3cp51387d

\section{Permanent link}

http://nrs.harvard.edu/urn-3:HUL.InstRepos:12748075

\section{Terms of Use}

This article was downloaded from Harvard University's DASH repository, and is made available under the terms and conditions applicable to Other Posted Material, as set forth at http:// nrs.harvard.edu/urn-3:HUL.InstRepos:dash.current.terms-of-use\#LAA

\section{Share Your Story}

The Harvard community has made this article openly available.

Please share how this access benefits you. Submit a story.

Accessibility 
Cite this: Phys. Chem. Chem. Phys., 2013, 15, 13462

Received 2nd April 2013,

Accepted 11 th June 2013

DOI: $10.1039 / c 3 c p 51387 d$

www.rsc.org/pccp

\title{
Interplay of chemical disorder and electronic inhomogeneity in unconventional superconductors
}

\author{
Ilija Zeljkovic and Jennifer E. Hoffman*
}

\begin{abstract}
Many of today's forefront materials, such as high- $T_{c}$ superconductors, doped semiconductors, and colossal magnetoresistance materials, are structurally, chemically and/or electronically inhomogeneous at the nanoscale. Although inhomogeneity can degrade the utility of some materials, defects can also be advantageous. Quite generally, defects can serve as nanoscale probes and facilitate quasiparticle scattering used to extract otherwise inaccessible electronic properties. In superconductors, nonstoichiometric dopants are typically necessary to achieve a high transition temperature, while both structural and chemical defects are used to pin vortices and increase critical current. Scanning tunneling microscopy (STM) has proven to be an ideal technique for studying these processes at the atomic scale. In this perspective, we present an overview of STM studies on chemical disorder in unconventional superconductors, and discuss how dopants, impurities and adatoms may be used to probe, pin or enhance the intrinsic electronic properties of these materials.
\end{abstract}

\section{Introduction}

Conventional superconductors exhibit two defining properties: (1) vanishing of electrical resistivity below a critical temperature

Department of Physics, Harvard University, Cambridge, MA 02138, USA.

E-mail: jhoffman@physics.harvard.edu

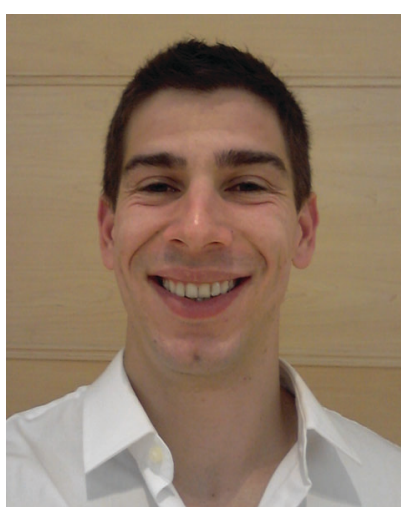

Ilija Zeljkovic
Ilija Zeljkovic is a Postdoctoral Research Fellow at Boston College. He earned his dual BSC degree in Physics and Computer Science from Washington University in Saint Louis in 2007, and received his $P h D$ in Physics from Harvard University in 2013. As a graduate student working in the laboratory of Prof. Jennifer Hoffman, he used scanning tunneling microscopy to investigate the interplay of electronic and structural properties in hightemperature superconductors. He is currently studying topological materials and complex oxide systems in the laboratory of Prof. Vidya Madhavan at Boston College.
$T_{\mathrm{c}}$, and (2) expulsion of magnetic flux below a critical field $H_{\mathrm{c}}$. The former was discovered by Kamerlingh-Onnes in $1911,{ }^{1}$ and the latter by Meissner and Ochsenfeld in $1933 .^{2}$ Almost 50 years after the initial discovery, Bardeen, Cooper and Schrieffer proposed a theory explaining the mechanism of superconductivity (BCS theory). ${ }^{3}$ According to BCS theory, a small attractive force between electrons, arising from their retarded interaction

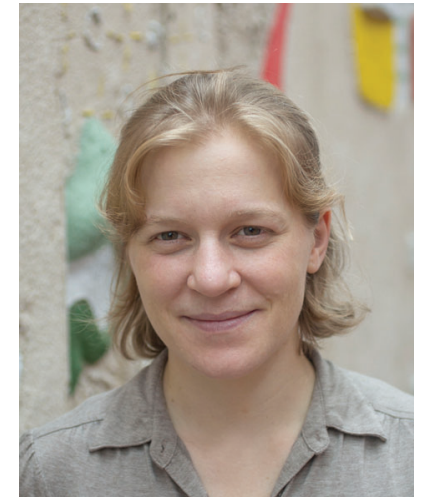

Jennifer E. Hoffman
Jennifer Hoffman is an Associate Professor of Physics at Harvard University. She earned her PhD in 2003 from the University of California, Berkeley, for the study of competing electronic orders in cuprate superconductors using scanning tunneling microscopy. As a postdoctoral researcher at Stanford University, she used magnetic force microscopy to investigate vortex pinning in superconductors. Since joining the faculty at Harvard, her research interests span a number of exotic materials, including unconventional superconductors, topological insulators, and strongly correlated materials such as vanadates. Her laboratory employs novel scanning probe techniques to image and manipulate nanoscale phase transitions in these materials. 
with the lattice (electron-phonon coupling), can pair the otherwise repulsive electrons, opening up a spectral gap in the electronic density of states (DOS) corresponding to the pairing energy. These "Cooper pairs" form an aggregate ground state which facilitates movement of charge through the crystal without loss of energy from scattering.

By the 1980s, superconductivity was considered theoretically solved but technologically marginal. McMillan had predicted the maximum $T_{\mathrm{c}}$ for phonon-induced Cooper pairing to be around $40 \mathrm{~K},{ }^{4}$ which is still inconveniently low for widespread commercial application. Then in 1986 came the discovery of an oxide superconductor with a $T_{\mathrm{c}}$ of $30 \mathrm{~K}$ (nominal composition $\left.\mathrm{Ba}_{x} \mathrm{La}_{5-x} \mathrm{Cu}_{5} \mathrm{O}_{5(3-y)}\right),{ }^{5}$ whose properties could not be explained by the BCS theory. Within one year, $T_{\mathrm{c}}$ in this new class of superconductors had already broken the liquid nitrogen barrier with the discovery of $\mathrm{YBa}_{2} \mathrm{Cu}_{3} \mathrm{O}_{7-x}$ (YBCO) with a $T_{\mathrm{c}}$ of $93 \mathrm{~K}^{6}$ Since the common property of all these materials was the layered crystal structure that contained one or more $\mathrm{CuO}_{2}$ planes, they were called "cuprates".

In contrast to the conventional superconductivity of elements, simple alloys, and stoichiometric compounds, high- $T_{\mathrm{c}}$ superconductivity in cuprates typically arises by off-stoichiometric doping of an antiferromagnetic parent compound. The parent compound is a Mott insulator due to the Coulomb repulsion that prevents double occupancy of $\mathrm{Cu}$ lattice sites, and splits the relevant $\mathrm{Cu} \mathrm{d}$ orbital into a filled lower Hubbard band (LHB) which is hybridized with the oxygen $\mathrm{p}$ orbitals and an empty upper Hubbard band (UHB). The bands are separated by a charge transfer gap of $\sim 2 \mathrm{eV}$. Upon the introduction of extra charge carriers into the system (hole or electron doping), the charges begin to hop between sites, states move into the gap, and the electronic properties of the material change dramatically (see Fig. 12a and b). ${ }^{7,8}$ As antiferromagnetism is weakened by doping, the material becomes superconducting, with $T_{\mathrm{c}}$ rising to a maximum and falling again, while the decreasing spectral gap indicates weakening pairing in the "overdoped" regime. Another prominent feature in the cuprate phase diagram is the mysterious "pseudogap" (PG) state characterized by the opening of a gap in the electronic DOS even above $T_{\mathrm{c}}$, predominantly on the underdoped side (Fig. 1a). Despite more than 25 years of cuprate research, neither the superconductivity nor the PG are well understood, and it is still debated whether the PG is a manifestation of a competing/coexisting order of a charge density wave (CDW) or a

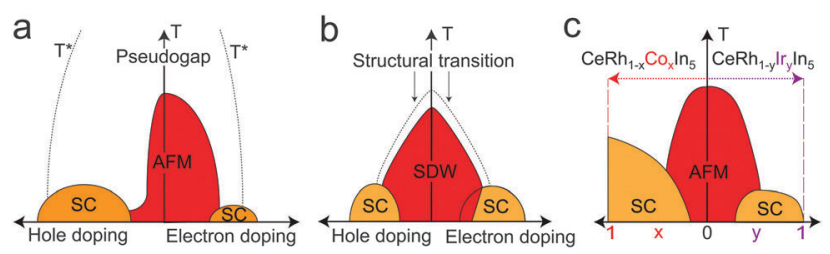

Fig. 1 Representative phase diagrams for (a) cuprates, (b) Fe-SCs, and (c) "heavyfermion" systems. Horizontal axis represents doping. SC, AFM and SDW represent superconducting, antiferromagnetic Mott insulating and spin density wave phases respectively. $T^{\star}$ denotes the temperature onset of the pseudogap phase in cuprates. ( $a$ and b) adapted from ref. 12. (c) adapted from ref. 19 for CeRhln ${ }_{5}$. spin density wave (SDW), ${ }^{9}$ or a Cooper-paired precursor to the superconducting state. ${ }^{10}$

The discovery of high- $T_{\mathrm{c}}$ superconductivity in Fe-based materials (Fe-SCs) in 2008 (ref. 11) provided a new opportunity for comparison with cuprates. ${ }^{12}$ Similar to cuprates, Fe-SCs exhibit a dome-shaped superconducting phase diagram with a layered crystal structure and an antiferromagnetic parent compound (Fig. 1b). However, the parent state antiferromagnetism is itinerant, and of a different ordering wavevector than in cuprates. Superconductivity can be induced or enhanced in Fe-based parent compounds by a wider variety of mechanisms, ${ }^{13,14}$ including electron or hole doping, chemical or external pressure, and apparently even treatment with alcoholic beverages. ${ }^{15}$ Almost five years since the initial discovery, the maximum $T_{\mathrm{c}}$ in bulk Fe-SCs remains capped at $\sim 57 \mathrm{~K}$ for $\mathrm{Sm}_{0.95} \mathrm{La}_{0.05} \mathrm{O}_{0.85} \mathrm{~F}_{0.15} \mathrm{FeAs}^{16}$ and $\sim 56 \mathrm{~K}$ for $\mathrm{Gd}_{1-x} \mathrm{Th}_{x} \mathrm{FeAsO},{ }^{17}$ while superconductivity up to $65 \mathrm{~K}$ has very recently been reported in single layer FeSe films. ${ }^{18}$

Unconventional superconductivity is also present in some of the strongly-correlated heavy-fermion materials (HFs). These materials contain elements with f-orbitals, whose localized spins interact with those of the itinerant electrons, generating exotic electronic states with effective electron mass several orders of magnitude higher than that of a bare electron. Furthermore, when the interaction between the f-orbital electrons and the conduction electrons is tuned by chemical or external pressure, HFs can superconduct with $T_{\mathrm{c}}$ on the order of several Kelvin (Fig. 1c). ${ }^{19}$ Because the velocity of the heavy quasiparticles is reduced by nearly three orders of magnitude, the retarded lattice interaction may no longer be able to overcome the repulsive electron interaction, which points instead towards unconventional pairing mechanisms involving higher angular momentum and spin states.

Despite concerted effort in the past few decades to further the understanding of high- $T_{\mathrm{c}}$ and HF materials, several of their properties remain puzzling. For example, cuprates are generally accepted to have a sign-changing $d$-wave pairing order parameter with four gap nodes, ${ }^{20}$ HF superconductors are thought to be similar, ${ }^{19}$ while many Fe-SCs seem to exhibit a nodeless $s_{ \pm}$symmetry $^{13,14}$ that is by no means universal. ${ }^{21}$ High- $T_{\mathrm{c}}$ superconductors are typically electronically inhomogeneous at the atomic scale, but to what extent the intrinsic chemical or structural disorder is responsible for electronic inhomogeneity, and whether the inhomogeneity is relevant to superconductivity, are unresolved questions. In contrast to bulk-probe techniques that measure average properties over large areas of the sample, scanning tunneling microscopy (STM) is a real-space technique able to measure the electronic density of states with atomic resolution. In this Perspective, we will discuss the impact of STM imaging studies of single atom defects in unconventional superconductors. We will begin with a description of how defects can be used to extract intrinsic properties of unconventional superconductors - as single atom probes, or as scattering centers to allow direct imaging of quasiparticle dispersion. We will then review how impurities may impact relevant material properties such as electronic inhomogeneity, critical temperature $T_{\mathrm{c}}$, and critical current $J_{\mathrm{c}}$. 


\section{Scanning tunneling microscopy and spectroscopy}

STM, pioneered by Binnig and Rohrer in $1982,{ }^{22}$ is based on the quantum tunneling of electrons between two electrodes separated by a potential barrier. The experimental setup consists of a sharp metallic tip (used as a local probe) which is brought within several $\AA$ of a conducting surface using a three-dimensional piezoelectric scanner (Fig. 2a). This scanner can position the tip both laterally (in the $x y$ plane) and vertically (in the $z$-direction) with sub-^ precision. Application of voltage between the tip and the sample allows electrons to quantum-mechanically tunnel between the two (Fig. 2b). The tunneling current generated is approximated by:

$$
I \propto \mathrm{e}^{-2 \kappa d}, \quad \kappa=\frac{\sqrt{2 m \phi}}{\hbar}
$$

where $d$ is the tip-sample separation and $\phi$ is a mixture of the work functions of the tip and sample. ${ }^{23}$

STM can reveal both the information about the geometry of the surface and the evolution of the DOS. The surface contour can be mapped based on the exponential dependence of the tunneling current $I$ on the tip-sample separation distance $d$. As the tip is rastered across the surface in the $x y$-plane at a fixed bias voltage $V$, the feedback loop adjusts the position of the tip in the $z$-direction to maintain the measured current $I_{\text {meas }}$ at a fixed setpoint value $I_{\text {set }}$ (Fig. 2a). In this technique, referred to as the constant-current topographic mode, the $z$ trajectory of the tip maps a contour of constant integrated density of states from the Fermi level to eV. For a sample with a homogeneous DOS, this contour corresponds purely to the geometric surface corrugations. However, many materials exhibit a spatially heterogeneous DOS, which means that STM topographs of these compounds represent a combination of effects due to geometric corrugations and electronic DOS.

Besides acquiring information about the surface geometry, STM can measure the electronic DOS at energies of up to several electron volts, both in occupied and unoccupied sample

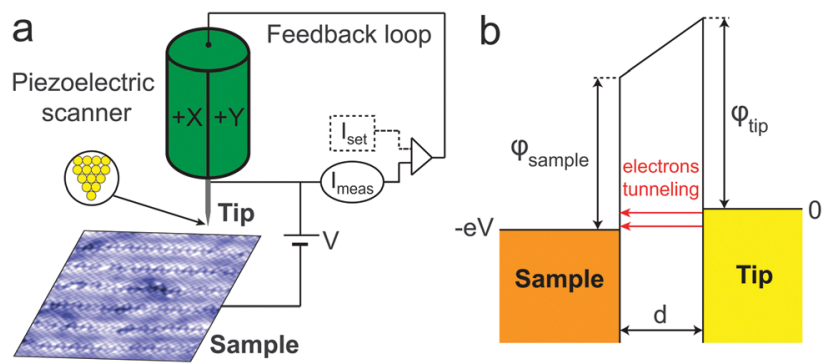

Fig. 2 Basic principles of STM and scanning tunneling spectroscopy (STS). (a) Schematic representation of STM. A voltage $V$ is applied between the tip and the sample. The tip is rastered across the surface in the $x y$ plane and its $z$ coordinate is adjusted using the three-dimensional piezoelectric scanner controlled by a feedback loop. (b) Quantum tunneling of electrons between the tip and the sample across a vacuum barrier of width $d$ upon the application of a voltage bias $V$ between the two. If a positive $V$ is applied to the sample, the Fermi level of the sample shifts down with respect to the Fermi level of the tip, and electrons tunnel from the occupied states of the tip into the empty states of the sample. states. The measurement is achieved by turning off the feedback loop (which fixes the tip-sample distance $d$ ), sweeping the bias voltage $V$, and measuring the current response $I(V)$. The conductance $\mathrm{d} I / \mathrm{d} V$ is usually measured using a lock-in amplifier technique, where a small bias voltage modulation $\mathrm{d} V$ (typically a few millivolts) is added to $V$, and the change in the tunneling current $\mathrm{d} I$ is measured to obtain $\mathrm{d} I / \mathrm{d} V$. The $\mathrm{d} I / \mathrm{d} V$ spectrum is to a good approximation directly proportional to the electronic DOS, with a multiplier that is spatially dependent (due to the "setup condition" which fixes the tip-sample distance $d$ ), but nearly energy independent. ${ }^{23}$ Furthermore, $\mathrm{d} I / \mathrm{d} V$ spectra acquired at a dense $x y$ grid of points allow a spatial visualization of the evolution of the DOS with energy (local DOS mapping).

\section{Defects as "nanoprobes"}

Crystal defects, whether native or intentionally introduced, can be used to probe intrinsic material properties. In the following sections, we will discuss several means by which single atomic defects can be used to measure the electronic properties of a material.

\section{Single atom defects as local probes}

While in conventional $s$-wave superconductors, only magnetic impurities break pairs, in an unconventional superconductor with a sign-changing order parameter, both magnetic and nonmagnetic impurities are expected to degrade superconductivity and induce states within the superconducting gap. ${ }^{24} \mathrm{~A}$ number of theoretical studies predicted that impurities in cuprates could have STM-observable spatial and spectroscopic signatures of the symmetry of the superconducting order parameter (OP) and even the mechanism of superconducting pairing. ${ }^{25-27}$ More recent work followed up with predictions about the effects of impurities in different order parameter scenarios in Fe-SCs. ${ }^{28-34}$

A bound state is a normalizable localized state in an attractive potential - typically decaying exponentially with distance from the potential minimum. For example, at defects or vortex cores of a conventional $s$-wave superconductor, bound states may occur with discrete energies less than the superconducting gap. However, in a superconductor with gap nodes, such states are not truly bound but "leak" along the nodal direction, with power-law decay that may not be normalizable. Such states are called virtual bound states. Quasiparticle scattering from real or virtual bound states, or even from repulsive potentials, may result in sub-gap states or "resonances" peaked at specific energies in the local density of states spectra acquired near the defect site. A dominant resonance below the Fermi level usually indicates an attractive potential, while a dominant resonance above the Fermi level indicates a repulsive potential. ${ }^{26}$

The family of Bi-based cuprates, first discovered in $1988,{ }^{35,36}$ boosted $T_{\mathrm{c}}$ up to $\sim 110 \mathrm{~K}$, and constituted the first generation of commercially produced high- $T_{\mathrm{c}}$ cables. ${ }^{37}$ Since adjacent BiO layers are weakly bonded by the van der Waals force, Bi-based cuprates typically cleave on a charge neutral plane between two 

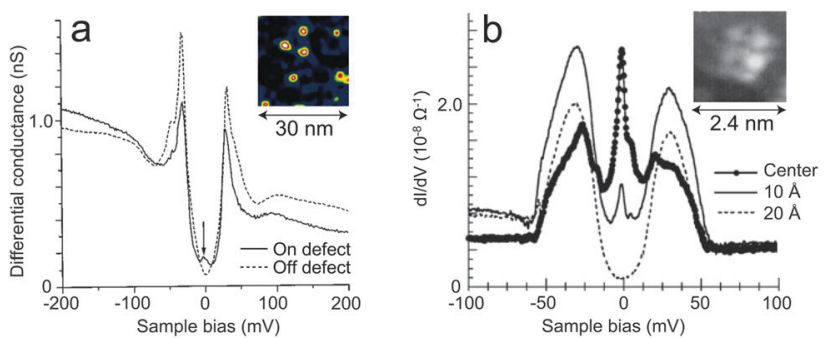

Fig. 3 Impurity-induced resonances in Bi-2212. (a) Typical d//dV spectra on and off a native defect of unknown origin. Inset shows a zero-bias conductance map of several such defects $(100 \mathrm{pA},-100 \mathrm{mV}, 4.2 \mathrm{~K}) .^{38}$ (b) d//dV spectra taken $10 \AA$ and $20 \AA$ away from the center of a cluster of Au atoms. Inset shows a topograph (200 pA, 32 mV, 5 K) of one such cluster. ${ }^{39}$

BiO layers (see the example of the BiO surface in Fig. 2a), and have thus been the main target among cuprates for STM studies. Pioneering studies of $\mathrm{Bi}_{2} \mathrm{Sr}_{2} \mathrm{CaCu}_{2} \mathrm{O}_{8+x}$ (Bi-2212) showed near-Fermi level resonances arising from intrinsic crystal defects $^{38}$ (Fig. 3a) and clusters of Au atoms deposited on the surface by an STM tip ${ }^{39}$ (Fig. 3b). The latter experiment, in which a sub-gap resonance arose from a non-magnetic impurity, supported a sign-changing $d$-wave order parameter.

As Bi-2212 crystal growth technology improved, samples with small numbers of controlled $\mathrm{Cu}$ site substitutions were investigated. First, non-magnetic Zn substitution in optimally doped Bi-2212 showed a sub-gap resonance arranged in a fourfold symmetric quasiparticle cloud aligned with the lattice (Fig. 4a).$^{40}$ The spectral signature of the $\mathrm{Zn}$ impurity state was particle-hole asymmetric - in fact, two orders of magnitude stronger at its main resonance energy of $-1.5 \mathrm{meV}$ than at the corresponding positive energy. Furthermore, the gap-edge peaks were almost completely destroyed within a $15 \AA$ radius of the $\mathrm{Zn}$ site, which the authors interpreted as local destruction of superconductivity. Hudson also imaged what they hypothesized to be vacancies at the $\mathrm{Cu}$ site (Fig. $4 \mathrm{~b}$ ). ${ }^{41}$ These vacancy defects were spatially and spectroscopically similar to the $\mathrm{Zn}$ defects, showing both an impurity-induced resonance at the Fermi level and the suppression of gap-edge peaks. The fact that $\mathrm{Zn}$ substitutions and native defects, both presumed to be non-magnetic impurities directly in the crucial superconducting layer, showed strong sub-gap resonances and superconductivity suppression, gave strong support to the sign-changing $d$-wave superconducting order parameter. However, Chatterjee et al. subsequently pointed out that features of similar shape in $\mathrm{Bi}_{2} \mathrm{Sr}_{2-y} \mathrm{La}_{y} \mathrm{CuO}_{6+x}(\mathrm{Bi}-2201)$ maintain their signature in $\mathrm{d} I / \mathrm{d} V$ well above the superconducting $T_{\mathrm{c}}$ (Fig. $4 \mathrm{c}$ ). ${ }^{42}$ Although unable to identify the chemical origin of these features, Chatterjee et al. concluded that the spatial and energy distribution of the impurity state is not determined by the superconducting gap, but rather by the PG.

In contrast to non-magnetic defects, substitution of magnetic $\mathrm{Ni}$ atoms at the $\mathrm{Cu}$ site in $\mathrm{Bi}-2212$ leads to a rather different behavior. ${ }^{43} \mathrm{Ni}$ atoms are magnetic, and thought to be in the $\mathrm{Ni}^{2+} 3 \mathrm{~d}^{8}$ electronic state with spin $S=1$, as compared to spin $S=\frac{1}{2}$ for $\mathrm{Cu}$ atoms. In $\mathrm{d} I / \mathrm{d} V$ images, $\mathrm{Ni}$ resonances are observed as "cross-shaped" features at $+9 \mathrm{meV}$
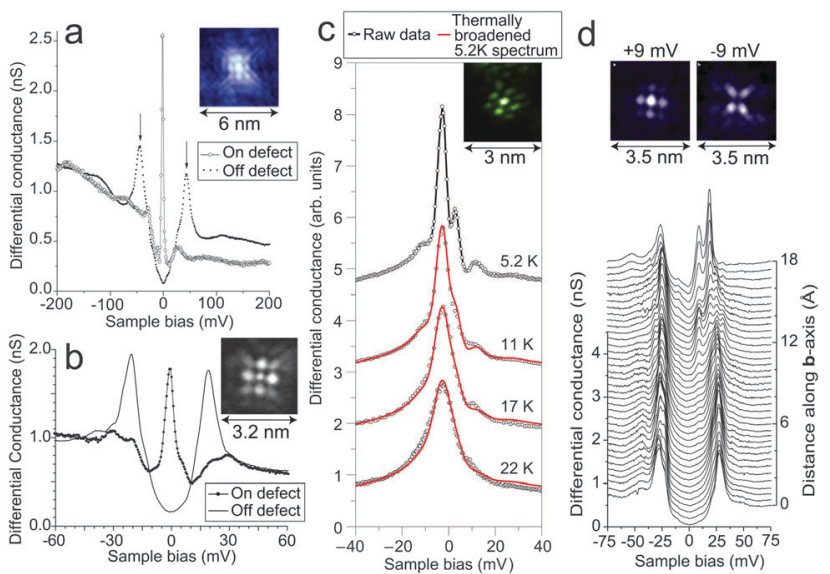

Fig. 4 Cu site defects in Bi-based cuprates. (a) $d / / d V$ spectra at and far away from a Zn substitution (200 pA, $-200 \mathrm{mV}$ ) in Bi-2212. Inset shows a single $\mathrm{Zn}$ impurity resonance in $d / / d V$ at $-1.5 \mathrm{mV}^{40}$ (b) Typical $d / / d V$ spectra at and far away from a presumed Cu site vacancy in Bi-2212 (200 pA, -200 mV, $4.2 \mathrm{~K}$ ). Inset shows a single vacancy resonance in $\mathrm{d} / / \mathrm{d} V$ at $-0.5 \mathrm{mV}$ bias. ${ }^{41}$ (c) Temperature evolution of $d / / d V$ spectra acquired at the center of an unknown impurity in Bi-2201 with $T_{\mathrm{c}} \sim 15 \mathrm{~K}$. For $\mathrm{d} / / \mathrm{d} V$ curves acquired above base temperature, the experimental data (black circles) are overlaid with red lines representing the $5.2 \mathrm{~K}$ spectrum thermally broadened to the respective temperatures. Inset shows a $d / /$ $\mathrm{d} V$ conductance map at $-2 \mathrm{mV}$ bias taken at $5.2 \mathrm{~K}$ on top of a single impurity. ${ }^{42}$ (d) Evolution of $\mathrm{d} / \mathrm{d} \mathrm{V}$ spectra as a function of distance away from a $\mathrm{Ni}$ substitution in Bi-2212, measured at $0.5 \AA$ intervals (100 pA, $-100 \mathrm{mV})$. Insets show $\mathrm{d} / / \mathrm{d} V$ maps on top of a $\mathrm{Ni}$ impurity. ${ }^{43}$

and as "X-shaped" features at $-9 \mathrm{meV}$ (inset in Fig. $4 \mathrm{~d}$ ). The spectral weight at the $\mathrm{Ni}$ impurity site spatially oscillates between two complementary components: particle-like (positivebias) and hole-like (negative-bias) (insets in Fig. 4d), but the spatially averaged spectrum of the impurity state is particle-hole symmetric. Furthermore, the magnitude of the presumed superconducting gap does not change as a function of distance away from the Ni site (Fig. 4d). This observation, combined with the particle-hole symmetric structure of the resonance, led Hudson et al. to conclude that magnetic Ni resonances do not disrupt superconductivity locally, in contrast to the destructive effect of non-magnetic $\mathrm{Zn}$. This suggests that the cuprate pairing mechanism is itself a magnetic one.

Although the cuprate order parameter was established as $d$-wave ${ }^{44}$ before single-atom imaging experiments became available, ${ }^{38,39}$ the order parameter in Fe-SCs has been much harder to pin down. Up to five bands cross the Fermi surface in Fe-SCs, allowing for many possible order parameters even within the same sub-family of these materials. ${ }^{21}$ Impurities may therefore be very important to gain insight into the pairing symmetry of these complex materials. ${ }^{28-34}$ Controlled studies can be carried out in the FeSe or LiFeAs families, which have mirror planes and are not subject to surface reconstructions. Furthermore, they are superconducting in stoichiometric form, so very sparse impurities can be studied in an otherwise pristine superconducting state.

Song et al. investigated single-atom impurities in FeSe films grown by molecular beam epitaxy (MBE), with $T_{\mathrm{c}} \sim 8 \mathrm{~K} \cdot{ }^{45}$ Both an Fe adatom evaporated onto the surface (Fig. $5 \mathrm{a}$ ) and a single 

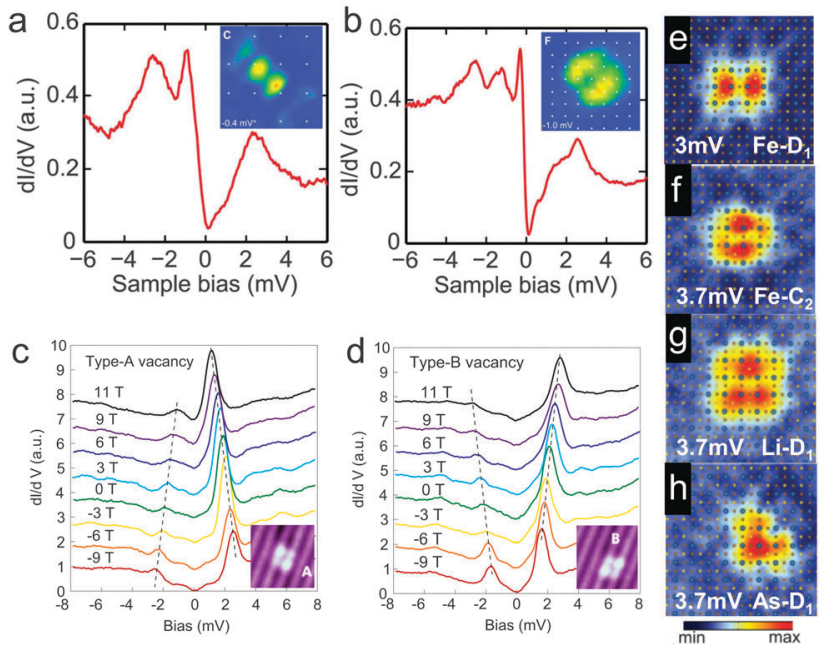

Fig. 5 Impurity-induced resonances in Fe-SCs. (a and b) d//dV spectra and maps of two defect types in FeSe (100 pA, $10 \mathrm{mV}, 0.4 \mathrm{~K}$ ). (a) d//dV on Fe adatom with map at $-0.4 \mathrm{mV}$; (b) $\mathrm{d} / / \mathrm{d} V$ on Se vacancy with map at $-1 \mathrm{mV}^{45}$ (c and d) $\mathrm{d} / / \mathrm{dV}$ spectra measured on top of two types of Fe vacancies in $\mathrm{KFe}_{2} \mathrm{Se}_{2}(100 \mathrm{pA}, 15 \mathrm{mV}$, $0.4 \mathrm{~K}$ ). Insets in (c) and (d) show real-space topographic images of the corresponding vacancies that appear as bright parallelograms of orthogonal orientation $(30 \mathrm{pA}, 30 \mathrm{mV}) .^{47} \mathrm{~d} / / \mathrm{d} V$ maps of $3.4 \mathrm{~nm}$ square areas of LiFeAs showing single defects at the (e and f) Fe site, ( $g$ ) Li site, and (h) As site, acquired at $3 \mathrm{mV}$, $3.7 \mathrm{mV}, 3.7 \mathrm{mV}$, and $3.7 \mathrm{mV}$ biases respectively, and $T=4.2 \mathrm{~K}$. Setup condition was $260 \mathrm{pA}$ and $25 \mathrm{mV}$ for $(\mathrm{e}-\mathrm{h}) .{ }^{46}$

Se vacancy (Fig. 5 b) induce an asymmetric, sub-gap resonance at small negative bias. The observed resonances are consistent with unconventional $s_{ \pm}$pairing. ${ }^{29,30}$

For nominally stoichiometric LiFeAs single crystals, Grothe et al. used STM to enumerate five distinct defect types of unknown origin, which can be classified based on their position and symmetry, all of which suppress the coherence peaks and induce sub-gap resonances at the impurity site. ${ }^{46}$ Only two of the defects, both identified to be at the Fe site, preserve the local symmetry of the lattice and have a single in-gap resonance (the $\mathrm{d} I / \mathrm{d} V$ spatial signature of one of them is shown in Fig. 5e). The other three defect types occur at the $\mathrm{Fe}, \mathrm{Li}$ and As sites (Fig. 5f-h), exhibit multiple sub-gap resonances, and break the local symmetry at the impurity site. All the observed sub-gap resonances are consistent with predictions for impurities in a superconductor with $s_{ \pm}$pairing symmetry. ${ }^{29,30}$

Although $\mathrm{KFe}_{2} \mathrm{Se}_{2}$ has no easy-cleave mirror plane like FeSe and LiFeAs, $\mathrm{KFe}_{2} \mathrm{Se}_{2}$ films can be grown by MBE with apparently unreconstructed 110 surfaces. ${ }^{47}$ Li et al. introduced Fe site defects, thought to be vacancies, into these films. The defects were imaged as bright parallelograms of two different configurations (Fig. $5 \mathrm{c}$ and d), both $C_{2}$ symmetric following the lattice symmetry of a single Fe vacancy. The $\mathrm{d} I / \mathrm{d} V$ spectra on top of each reveal (1) strong suppression of coherence peaks, consistent with the destruction of superconducting pairing through spin-flip scattering, and (2) a pair of sub-gap resonances at $\pm 1.9 \mathrm{mV}$ bias, symmetric around the Fermi level but with differing amplitudes attributed to the on-site Coulomb repulsion (Fig. $5 \mathrm{c}$ and d). These sub-gap resonances are consistent with both $s_{ \pm}$and $s_{++}$pairing symmetries. ${ }^{30}$ Because the energies of the resonances shift in opposite directions with applied field for the two types of vacancies, Li et al. concluded that the two vacancies carry different effective spin orientations. ${ }^{47}$ This discovery points towards a magnetically-related bipartite order in the low-temperature Fe lattice, which may suggest that $\mathrm{KFe}_{2} \mathrm{Se}_{2}$ is also orthorhombic, although direct structural evidence is lacking.

In addition to inducing sub-gap resonances which provide evidence for a sign flip of the superconducting order parameter in Fe-SCs, the spatial signature of defects may break the lattice symmetry and signal the presence of other symmetry-breaking orders. For example, in FeSe, both Fe adatoms and Se vacancies show strong rotational symmetry breaking, exhibiting dramatic two-fold symmetric electronic signatures, although their structural environment is four-fold symmetric to within $\sim 0.5 \%$. This observation is consistent with the elongated vortices seen in the same material ${ }^{45}$ and suggests two-fold symmetry of the gap function, possibly arising from orbital-ordering. ${ }^{48,49}$

In the same FeSe thin films, excess Se atoms substituting at the Fe sites (Fig. 6a) dramatically break the approximate $C_{4 \mathrm{v}}$ structural symmetry on two different length scales: (1) on the atomic scale where they take two different, orthogonally-oriented, dumbbell-shaped configurations, and (2) on a larger scale where they induce a $C_{2 \mathrm{v}}$-symmetric depression in the density of states centered at a single substitution (Fig. 6a). ${ }^{450}$ The smaller dumbbellshaped signature is present in topographs taken up to $3.5 \mathrm{~V}$ imaging bias, but the larger, $\sim 16 a_{\mathrm{Fe}-\mathrm{Fe}}$ sized dimers only appear at around $\pm 20 \mathrm{mV}$ bias, pointing towards their electronic origin. Furthermore, the larger features are always oriented along the orthorhombic a (longer, antiferromagnetic) $\mathrm{Fe}-\mathrm{Fe}$ axis, as they change their orientation by $90^{\circ}$ across an orthorhombic twin boundary, regardless of the stochastic orientation of the smaller dumbbell-shaped signature. They are qualitatively similar to the unidirectional nanostructures of size $\sim 8 a_{\mathrm{Fe}-\mathrm{Fe}}$ which are inferred to arise from Co dopants in lightly-doped (non-superconducting) $\mathrm{Ca}\left(\mathrm{Fe}_{1-x} \mathrm{Co}_{x}\right)_{2} \mathrm{As}_{2}$ (Fig. 6b); these are also inferred to change their orientation across an orthorhombic twin boundary. ${ }^{51}$ In contrast to the large electronic dimers around Fe site defects in FeSe (Fig. 6a), no large electronic dimer structure
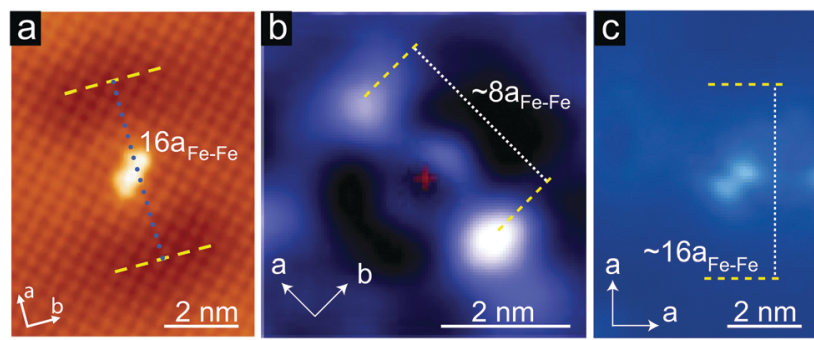

Fig. 6 Dimer impurity signatures in Fe-SCs. (a) A single Se substitution at a Fe site in the surface layer of a FeSe thin film, appearing as a small bright dumbbell along the Fe-As direction. The Fe site defect is surrounded by a larger electronic dimer structure: a $C_{2 v}$-symmetric depression in $d / / d V$ oriented along the Fe-Fe direction (100 pA, $10 \mathrm{mV}) .{ }^{50}$ (b) Average $\mathrm{d} / / \mathrm{d} V$ centered at a Co substitution at a Fe site in $\mathrm{Ca}\left(\mathrm{Fe}_{1-x} \mathrm{CO}_{x}\right)_{2} \mathrm{As}_{2}$ showing a similar large electronic dimer structure. ${ }^{76}$ (c) Topograph of an impurity in nominally stoichiometric LiFeAs showing the smaller dumbbell shape, but lacking the larger electronic dimer (10 pA, $20 \mathrm{mV}){ }^{53}$ 
has been observed at the impurities at the two inequivalent $\mathrm{Fe}$ sites in LiFeAs (Fig. 6c). This lack of observed larger electronic features in LiFeAs suggests that neither orbital-ordering nor pocket density wave ordering ${ }^{52}$ occur in a tetragonal Fe-SC.

We mention one final example where single atom impurities can be used to calibrate the STM technique itself. We note that recent breakthroughs in STM analysis allow for the removal of picoscale thermal drift and consequent improvement in image resolution. ${ }^{54,55}$ Such unit cell registry has enabled the claim of an intra-unit-cell nematic order ${ }^{54}$ and the understanding of the role of a subtle picoscale structure distortion. ${ }^{55}$ However, some doubts lingered about the registry between the topographic data used to remove the drift and the simultaneous conductance data used to draw conclusions about new phases. Hamidian et al. used the resonances from single $\mathrm{Zn}$ atoms in Bi-2212 to prove that the alignment between topographic and conductance data is accurate to better than $\sim 2 \%$ of a unit cell. ${ }^{56}$

In summary, we have given examples of the use of specific single atom impurities in unconventional superconductors to probe intrinsic material properties. In Bi-based cuprates, the spectral and spatial signature of defects at the $\mathrm{Cu}$ site confirmed $d$-wave pairing symmetry; or conversely, it has been confirmed that non-magnetic impurities in a sign-changing $d$-wave superconductor suppress coherence peaks and induce sub-gap states. In Fe-SCs, spectroscopy of all observed impurities (Fe and Se site defects in FeSe; Fe site defects in $\mathrm{KFe}_{2} \mathrm{Se}_{2}$; five types of defects in LiFeAs) shows coherence peak suppression and sub-gap resonances, pointing towards a sign-changing order parameter. Furthermore, the spatial signatures of the observed impurities in orthorhombic FeSe and $\mathrm{Ca}\left(\mathrm{Fe}_{1-x} \mathrm{Co}_{x}\right)_{2} \mathrm{As}_{2}$ show dramatic $C_{4}$ to $C_{2}$ symmetry breaking much more extreme than would be expected from the $\sim 0.5 \%$ structural orthorhombicity alone, providing evidence of orbital-ordering or other symmetry-breaking electronic states in orthorhombic Fe-SCs, in contrast to tetragonal LiFeAs. ${ }^{57}$

\section{Quasiparticle interference}

In addition to real-space studies of single point defects, STM can also be used as a probe of momentum space by quasiparticle interference (QPI) imaging. In a pioneering study, Crommie et al. observed quasiparticle standing waves scattering off step edges and point defects in $\mathrm{Cu}(111)$ (Fig. 7a). ${ }^{58}$ Analogous to throwing a rock into a pond and using the velocity and wavelength of resultant surface ripples to infer the depth of the pond and viscosity of the fluid, placing a defect into a material causes elastic scattering and interference of quasiparticles which can be used to extract the band structure of the material.

Elastic scattering mixes degenerate states with momenta $\vec{k}_{1}$ and $\vec{k}_{2}$, resulting in a standing wave of wavevector $\vec{q}=\vec{k}_{1}-\vec{k}_{2}$ in the DOS at energy $\varepsilon\left(\vec{k}_{1}\right)=\varepsilon\left(\vec{k}_{2}\right)$. Although some such standing waves may be observable in real space, the wavevectors of multiple coexisting standing waves arising from a complex band structure can be best quantified by Fourier transforming the real space image and fitting the peaks in $q$-space. The dominant wavevectors in $q$-space may typically be interpreted
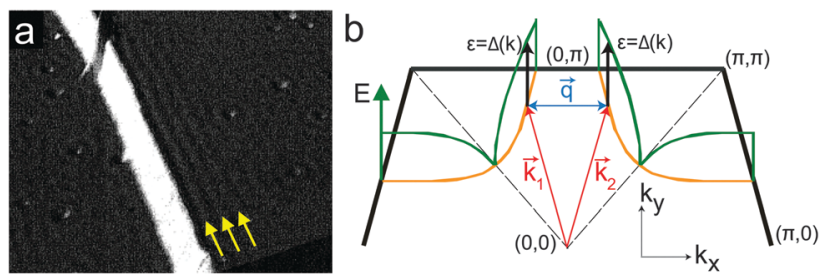

Fig. 7 Quasiparticle interference (QPI). (a) STM topograph showing standing wave patterns in the local density of states on a Cu(111) surface (100 mV, $1 \mathrm{nA}$ ). These spatial oscillations (exemplified by yellow arrows) represent quantummechanical interference patterns induced by quasiparticle scattering off step edges and surface defects. ${ }^{58}$ (b) Half of the Brillouin zone in Bi-2212. The superconducting energy gap $\Delta(\vec{k})$ (green) is shown along the normal state Fermi surface (orange). Interference patterns seen in STM images occur due to elastic scattering between regions of the same $\Delta(\vec{k})$, but different $\vec{k}$ (e.g. $\vec{k}_{1}$ and $\vec{k}_{2}$ ), resulting in a wavevector $\vec{q}=\vec{k}_{1}-\vec{k}_{2}$ (adapted from ref. 62 ).

as arising from regions of high joint density of states (JDOS) in $k$-space. For example, the $d$-wave superconducting gap of Bi-2212 produces an octet of high-DOS regions for each energy, on the locus of the normal state Fermi surface, ${ }^{59-61}$ two of which are labeled by $\varepsilon=\Delta(k)$ in Fig. $7 \mathrm{~b}$. Using the symmetries of the Brillouin zone, the original $\vec{k}_{1}$ and $\vec{k}_{2}$ on the Fermi surface can be determined from the observed wavevector $q$ at a given energy $\varepsilon$. Repeating this process for different energies maps the contour of the Fermi surface. Additionally, this method can be used to trace the momentum-dependence of the superconducting gap $\Delta(k)=\varepsilon(q)$ along the Fermi surface.

QPI imaging has subsequently played a pivotal role in the study of unconventional superconductors and other materials in which strong correlations necessitate both real-space and momentum-space mapping for complete understanding. Dopants or naturally occurring defects serve as sufficient scatterers for QPI imaging in many of the unconventional superconductors discussed here, but in some cases scatterers must be intentionally introduced into otherwise clean materials in order to access the crucial information that QPI imaging can provide. In the following section, we will discuss the relation between specific impurities and the observation of QPI in a variety of unconventional superconductors: cuprates (Bi-2212, $\mathrm{Na}_{x} \mathrm{Ca}_{2-x} \mathrm{CuO}_{2} \mathrm{Cl}_{2}$ (Na-CCOC)), Fe-SCs (LiFeAs, $\mathrm{Ca}\left(\mathrm{Fe}_{1-x} \mathrm{Co}_{x}\right)_{2} \mathrm{As}_{2}$ ) and $\mathrm{HF}$ compounds $\left(\mathrm{URu}_{2} \mathrm{Si}_{2}, \mathrm{CeCoIn}{ }_{5}\right)$.

Bogoliubov QPI patterns (interference of superconductor quasiparticles) were first imaged ${ }^{59}$ and theoretically explained ${ }^{60}$ a decade ago in Bi-2212, providing a quantitative measure of the angle dependence of the $d$-wave order parameter. ${ }^{61}$ Subsequent work has used QPI to match the electronic band structure of Bi-2212 to that determined by angle-resolved photoemission spectroscopy (ARPES), thus providing support for the sensitivity of both surface measurement techniques to intrinsic properties. ${ }^{63-65}$

In the last decade, QPI has enabled many discoveries in cuprates. For example, QPI was utilized to observe the "extinction" of Bogoliubov quasiparticles in Bi-2212 at the antiferromagnetic Brillouin zone edge, with implications for the role of the PG or other electronic reconstructions there. ${ }^{66}$ It was also used to enable mapping of the nanoscale variations in the band structure of Bi-2201, highlighting the extreme effects of strong 
correlations in these materials. ${ }^{67}$ QPI can even be used as a phase-sensitive technique, e.g. to extract the $d$-wave coherence factors of Na-CCOC. ${ }^{68}$ Recently, He et al. used doping and magnetic field dependence of QPI to prove that $d$-wave Bogoliubov quasiparticles exist on the antinodal part of the Fermi surface, and to identify the competitive nature of superconductivity and the pseudogap. ${ }^{69}$

Several possible defects have been suggested as the primary scattering sites responsible for this wealth of information from QPI in Bi-2212: interstitial O atoms, $\mathrm{Sr}$ or $\mathrm{Cu}$ site defects, and 2-3 nm sized "patches" of different spectral gaps. However, no consensus has been reached. Although it was claimed that some interstitial $\mathrm{O}$ atoms were correlated with the minima of the observed QPI patterns,$^{70}$ which might suggest that these dopants act as scattering sites, it was later noted ${ }^{71}$ that the correlation was more likely to be related to a static charge order and not the dispersive QPI. In our recent experiments on the latest generation of Bi-2212 samples grown by Gu et al. ${ }^{71}$ with $T_{\mathrm{c}}$ ranging from $55 \mathrm{~K}$ underdoped to $91 \mathrm{~K}$ optimally doped, we have rarely observed QPI, thus making O disorder, $\mathrm{Sr}$ site defects, and spectral gap "patches" (all present in our samples) unlikely candidates for the QPI source. We also found far fewer low energy resonances from native $\mathrm{Cu}$ site defects in these newer samples compared to earlier samples, ${ }^{41,62}$ which points towards these intrinsic low energy defects, thought to be $\mathrm{Cu}$ vacancies ${ }^{41}$ as the strongest candidates for scattering sites in Bi-2212. This suggests that such defects which occurred accidentally in early generation Bi-2212 samples may need to be intentionally reintroduced in order to continue to study QPI in cleaner new Bi-2212 samples.

QPI imaging has recently been extended to several Fe-SCs. Notable results include evidence for nematicity in the $\mathrm{CaFe}_{2} \mathrm{As}_{2}$ parent compound, ${ }^{51}$ evidence for $s_{ \pm}$pairing from QPI coherence factors in $\mathrm{FeSe}_{1-x} \mathrm{Te}_{x},{ }^{7-74}$ and quantitative extraction of the gap anisotropy on the multiple Fermi sheets of LiFeAs. ${ }^{75}$ Chuang et al. used QPI to infer a nematic electronic structure in lightly-doped (non-superconducting) $\mathrm{Ca}\left(\mathrm{Fe}_{1-x} \mathrm{Co}_{x}\right)_{2} \mathrm{As}_{2}$ (Fig. 8a and $\mathrm{b}),{ }^{51}$ comprised of electronic dimers oriented along the $a$ (longer, antiferromagnetic) $\mathrm{Fe}-\mathrm{Fe}$ axis and centered at the $\mathrm{Co}$ dopants. ${ }^{76}$ No such electronic dimers were observed in nominally stoichiometric $\mathrm{CaFe}_{2} \mathrm{As}_{2}$, supporting the essential role of Co atoms for QPI in $\mathrm{Ca}\left(\mathrm{Fe}_{1-x} \mathrm{Co}_{x}\right)_{2} \mathrm{As}_{2}$ and possibly explaining the transport anisotropy observed in this material. ${ }^{76}$ In contrast to $\mathrm{Ca}\left(\mathrm{Fe}_{1-x} \mathrm{Co}_{x}\right)_{2} \mathrm{As}_{2}$, LiFeAs is both nominally stoichiometric and superconducting. Allan et al. used intraband QPI in this Fe-SC to quantify the anisotropic superconducting energy gap $\Delta_{k}$. Although nominally stoichiometric, LiFeAs topographs display several types of native defects (Fig. 8c and 5e-h) which may induce QPI scattering shown in Fig. 8d, but the exact identity of these defects remains unknown.

QPI imaging has been utilized to reveal the electronic structure of $\mathrm{HF}$ superconductors above $T_{\mathrm{c}},{ }^{77-80}$ and very recently below $T_{\mathrm{c}}{ }^{81}$ Above $T_{\mathrm{c}}$, the normal state electronic properties of these materials are dominated by the hybridization of the conduction band (typically a light band of s, p, and/ or d character), with localized f orbitals, resulting in heavy,
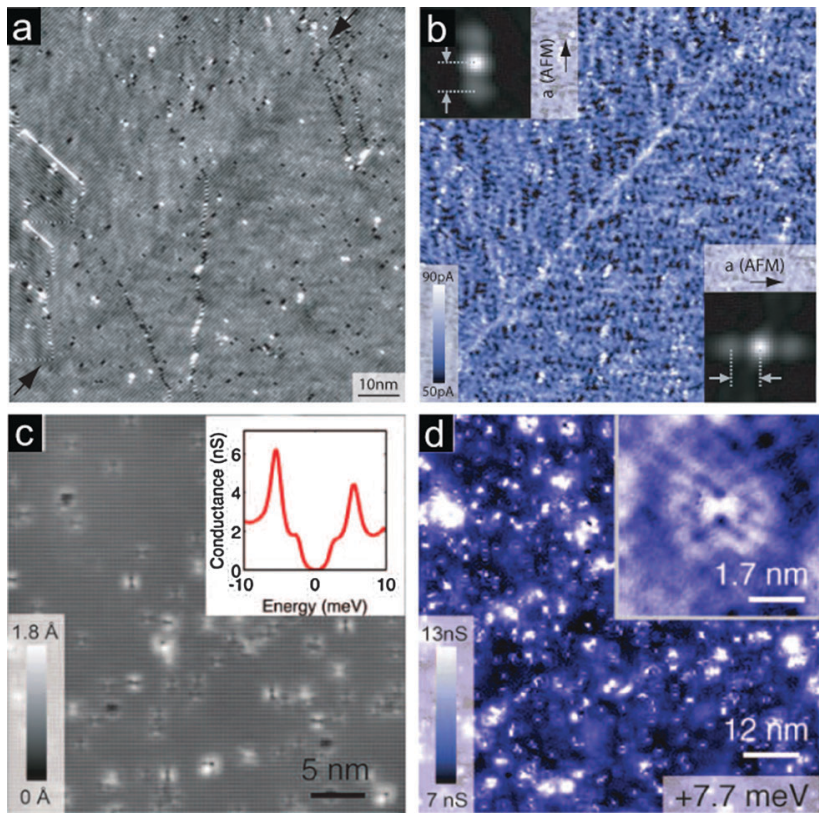

Fig. 8 QPI studies in Fe-SCs. (a) Simultaneous topograph and (b) integral of $\mathrm{d} / / \mathrm{d} V$ from Fermi level to $50 \mathrm{mV}$ of lightly doped non-superconducting $\mathrm{Ca}\left(\mathrm{Fe}_{1-x} \mathrm{Co}_{x}\right)_{2} \mathrm{As}_{2}$ with $x \sim 3 \%(100 \mathrm{pA},-50 \mathrm{mV}) .^{51}$ Dimer features in (b) rotate by $\sim 90^{\circ}$ across an orthorhombic twin boundary. (c) $A \sim 35 \mathrm{~nm}$ topograph image of nominally stoichiometric LiFeAs showing various intrinsic defects as bright features. Inset in (c) shows an average $d / / d V$ spectrum away from impurities. ${ }^{75}$ (d) $\mathrm{d} / / \mathrm{d} V(7.7 \mathrm{mV}$ ) on LiFeAs showing an intraband QPI scattering pattern. Inset in (d) shows typical QPI oscillations surrounding an impurity atom. $^{75}$

hybrid bands as sketched in Fig. 9a. For example, $\mathrm{URu}_{2} \mathrm{Si}_{2}$ begins to exhibit heavy fermion properties at $\sim 55 \mathrm{~K}$, goes through a "hidden order" transition at $T_{0} \sim 17.5 \mathrm{~K}$, and eventually becomes a bulk superconductor at $T_{\mathrm{c}} \sim 1.5 \mathrm{~K} .{ }^{82}$ Schmidt et al. employed QPI imaging to track the hybridization through the notorious "hidden order" transition, using $1 \%$ non-magnetic Th substituted at the U sites to yield QPI patterns without otherwise altering the properties of the pure material. ${ }^{77}$ Hamidian et al. further revealed oscillations in the hybridization strength originating at each $\mathrm{Th}$ atom, which acts as a single Kondo hole. ${ }^{79}$ In the CeMIn ${ }_{5}$ family of HFs (Fig. 1c), $0.15 \% \mathrm{Hg}$ substitutions at the In lattice sites of $\mathrm{CeCoIn}_{5}$ served a similar purpose ${ }^{80}$ providing the scattering necessary for QPI, without changing the intrinsic properties of the material. ${ }^{83}$ Aynajian et al. used the energy dispersion of the QPI wavevectors to track the increase in effective electron mass through the gradual hybridization around $50 \mathrm{~K}$.

Recently, Allan et al. performed low temperature studies down to $0.25 \mathrm{~K}$ to complete the picture of HF superconductivity in $\mathrm{CeCoIn}_{5}$. In addition to QPI observed at energy scales of $\pm 100 \mathrm{meV}$ from the conventional bands, and at $\pm 15 \mathrm{meV}$ from the hybridized heavy bands (sketched in Fig. 9b), Allan et al. revealed Bogoliubov QPI patterns related to superconductivity within the energy range of $\pm 1 \mathrm{meV} .{ }^{81}$ The closing of the low temperature spectral gap of $\sim 550 \mu \mathrm{eV}$ at the bulk $T_{\mathrm{c}}=2.1 \mathrm{~K}$, along with images of a square Abrikosov vortex lattice, consistent with that reported by neutron scattering experiments, ${ }^{84}$ 

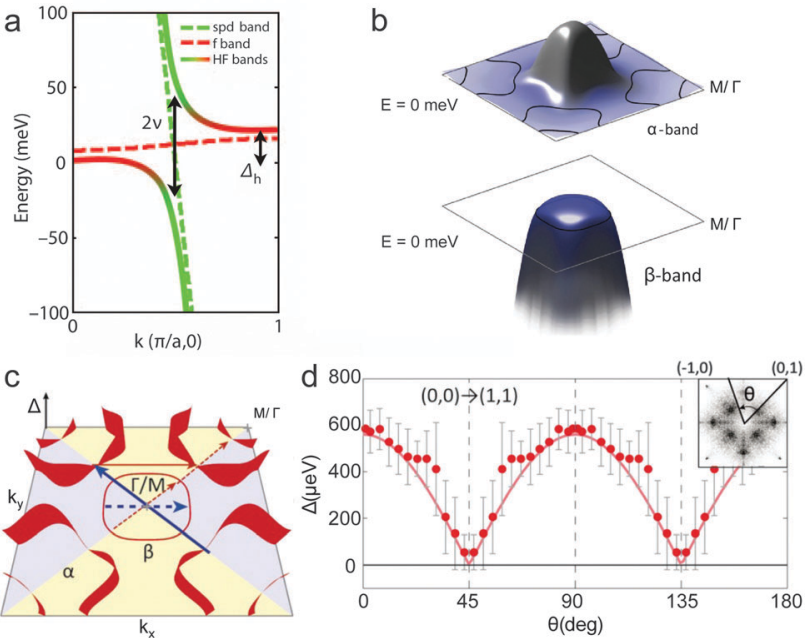

Fig. 9 (a) Sketch of the generic bare electronic bands (dashed lines) and hybridized heavy fermion bands (solid lines) displaying a direct $(2 \nu)$ and an indirect $\left(\Delta_{\mathrm{h}}\right)$ hybridization gap. ${ }^{80}$ (b) Details of the hybridized bands of CeColn $\mathrm{n}_{5}$ in 2-dimensional $k$-space, with the Fermi surface shown as black lines. (c) The modeled energy gap magnitudes $\Delta_{i}(k)$ used to achieve the most successful QPI simulations are shown in red on the three Fermi surfaces. (d) Measured $\Delta(\theta)$ and its comparison with the simplest multi-band gap structure $\Delta_{\beta}(k)=0$ and $\Delta_{\alpha}(k)=$ $A \cos (2 \theta) ; A=550 \mu \mathrm{eV}$ which is found to be consistent with the QPI data. ${ }^{81}$

confirmed the observation of superconductivity in $\mathrm{CeCoIn}_{5}$. Furthermore, the V-shaped spectra suggested gap nodes, while the QPI patterns imaged (Fig. 9d inset) were most consistent with $\mathrm{d}_{x^{2}-y^{2}}$ gap symmetry, and allowed the first mapping of the momentum-space structure of the superconducting energy gap $\Delta(k)$ in any HF compound (Fig. 9c and d).

In summary, we have emphasized the utility of QPI imaging to reveal important information about various electronic states in unconventional superconductors. In cuprates, Fe-SCs, and HF materials, QPI has been used to extract the band structure and angular dependence of the superconducting gap, as well as to provide constraints on the pairing symmetry. Defects are a prerequisite for all such studies. In some cases (e.g. $\mathrm{URu}_{2} \mathrm{Si}_{2}$ and $\operatorname{CeCoIn}_{5}$ ), such defects must be intentionally introduced in order to gain the desired information. In other cases (e.g. Bi-2212 and LiFeAs), native scatterers are often present, but their identity remains unknown. Based on previous studies and on our experimental data, intrinsic $\mathrm{Cu}$ site vacancies are likely candidates for the QPI scattering sources in Bi-2212. We suggest that such defects may need to be intentionally re-introduced into the new, cleaner generation of $\mathrm{Bi}-2212$ samples that seem to lack observable QPI patterns.

\section{Chemical disorder as a tuning parameter}

Most high- $T_{\mathrm{c}}$ superconductors exhibit a significant amount of chemical disorder since isovalent or aliovalent substitutions, lattice site vacancies, or interstitial atoms are often required to achieve superconductivity. What influence does this chemical inhomogeneity have on the local and global electronic properties? In the following sections, we will discuss how the concentration of specific dopants and their spatial distributions may affect the local electronic inhomogeneity as well as some of the most prominent global properties of superconductors crucial for applications.

\section{Relationship between chemical disorder and electronic inhomogeneity in cuprates}

The earliest and highest- $T_{\mathrm{c}}$ cuprates have been chemically nonstoichiometric. In parallel, electronic inhomogeneity has been an essential component to theories of high- $T_{\mathrm{c}}$ since the beginning. An obvious question is: to what extent are these related and to what extent can chemical inhomogeneity drive electronic inhomogeneity which directly enhances $T_{\mathrm{c}}$ ?

Several types of nanoscale electronic inhomogeneity have been theoretically predicted, ${ }^{85-88}$ and experimentally observed by STM ${ }^{67,89-96}$ in many cuprate superconductors. We categorize the inhomogeneity as follows. Type I inhomogeneity refers to the variation of the prominent spectral gap by a factor of $\sim 2$ on 2-3 nm length scales. Although this variation was initially attributed to the superconducting gap, ${ }^{89,90,92,94}$ more recent work attributes the dominant variation to the PG. ${ }^{96,97}$ Type II refers to "checkerboard" (CB) charge order that appears as a spatial modulation of spectral weight most noticeable around the Fermi energy. It is disordered and possibly associated with the PG state. ${ }^{67,93,94,98-100}$ However, the exact relationship between the nanoscale electronic inhomogeneity and chemical disorder remains unresolved. In this section we summarize STM experiments on several cuprates (Bi-2212, Na-CCOC and YBCO) with bearing on the following controversy - is intrinsic electronic inhomogeneity in cuprates spontaneously arising (and pinned by chemical inhomogeneity), or is it directly caused by chemical inhomogeneity?

Bi-2212. STM topographs of the cleaved surface of Bi-2212 (crystal structure and cleavage plane shown in Fig. 10a) reveal a clear Bi lattice, as well as bright and dark patches arising from spectral inhomogeneity (Fig. 10b). Bi-based cuprates are nonstoichiometric, with several candidate dopants, vacancies, and substitutions possibly responsible for spectral inhomogeneity. Here we will discuss the effects of $\mathrm{O}$ interstitials and vacancies, as well as substitutions at the $\mathrm{Bi}, \mathrm{Sr}$, and $\mathrm{Cu}$ sites, in $\mathrm{Bi}-2212$.

Oxygen doping of at least $5 \%$ is necessary to achieve superconductivity in Bi-2212 (the optimal concentration is 16\%). The random spatial distribution of interstitial oxygen dopants is an obvious candidate to cause type I inhomogeneity. ${ }^{86}$ However, this hypothesis was initially difficult to test since typical, lowenergy STM experiments were unable to locate these dopants, and more challenging experiments at higher energies were needed. In a pioneering study, McElroy et al. imaged a set of interstitial oxygen atoms in $\mathrm{Bi}-2212$ as atomic-scale resonances in $\mathrm{d} I / \mathrm{d} V$ images at $-0.96 \mathrm{~V}^{\text {bias }^{70}}$ (later named type-B oxygens ${ }^{101}$ ). However, the correlation between the locations of the observed $\mathrm{O}$ dopants and the magnitude of the spectral gap was relatively weak and of unexpected sign: although it is well-established that the dominant spectral gap, now known to be the PG, decreases monotonically with increasing $\mathrm{O}$ dopant concentration, ${ }^{9} \mathrm{McEl}$ roy's O dopants were more likely to be found in the regions of high PG. 
a

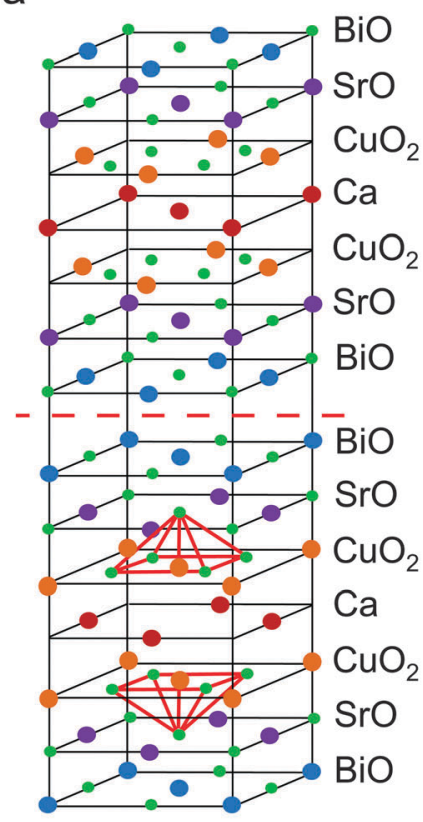

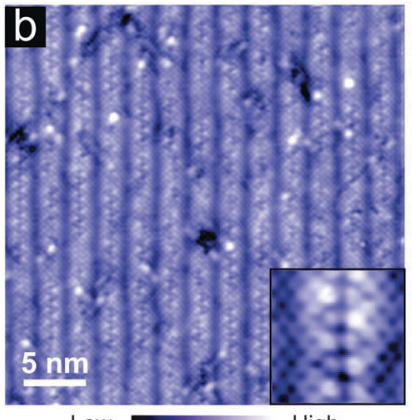

Low

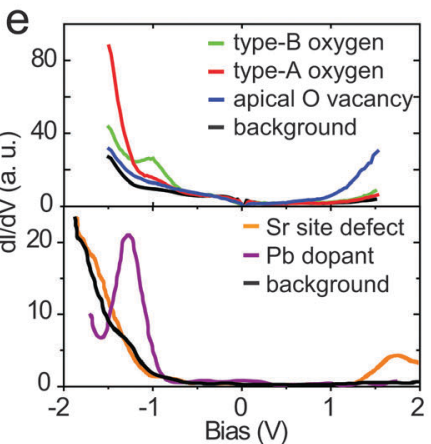

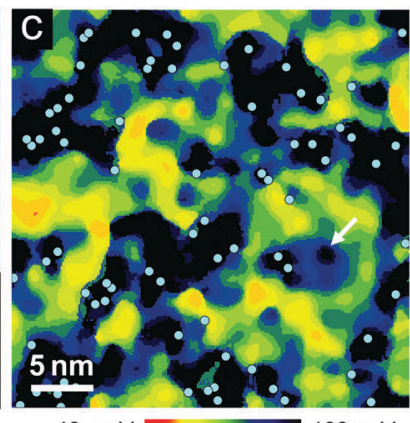

$40 \mathrm{meV} 100 \mathrm{meV}$

f 0.50

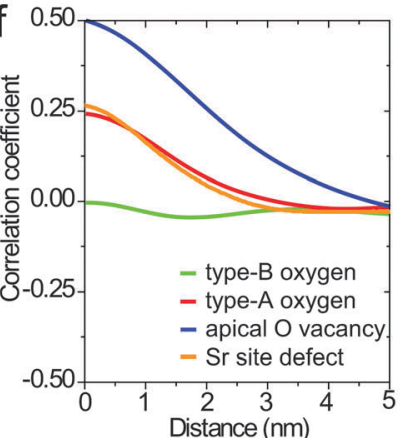

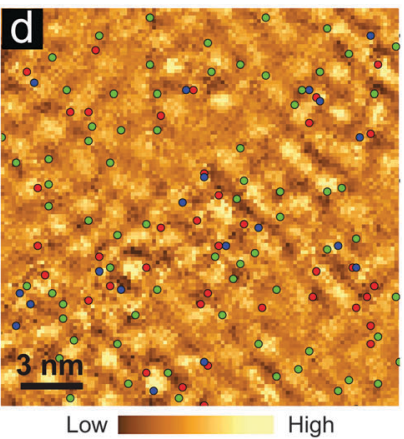

9

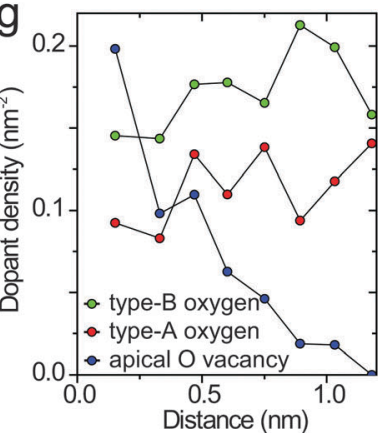

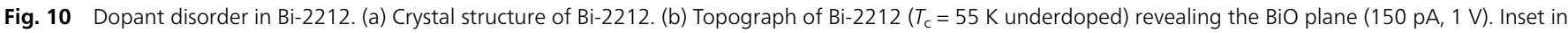

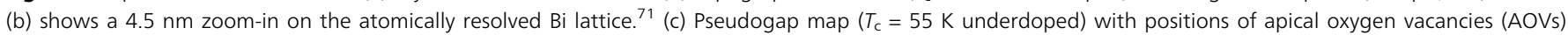

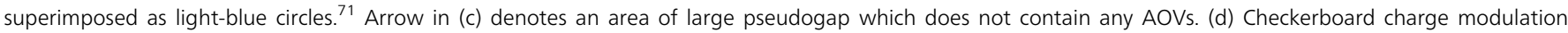

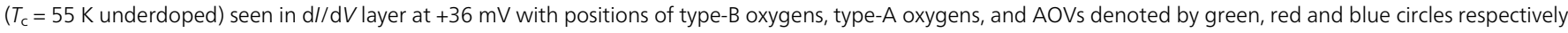

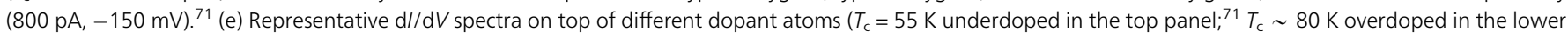

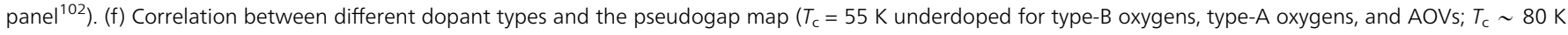

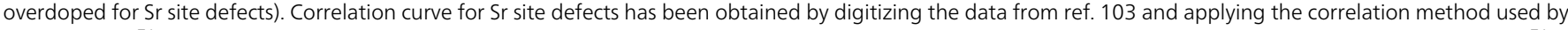
Zeljkovic et al. ${ }^{71}$ for direct comparison. (g) Density of each type of O defect as a function of distance from the nearest "checker" peak ( $T_{\mathrm{c}}=55 \mathrm{~K}$ underdoped). ${ }^{71}$

In order to address the surprising correlation, a theoretical study proposed the existence of a second set of interstitial $\mathrm{O}$ dopants (type-A oxygens) which would reside closer to the $\mathrm{CuO}_{2}$ plane compared to type-B oxygens. ${ }^{101}$ The type-A oxygens were predicted to have a greater effect on the $\mathrm{CuO}_{2}$ plane and thus resolve the surprising weak positive correlation between type-B O locations and PG magnitude. ${ }^{101}$ By extending the energy range of local spectroscopy on Bi-2212 even further than in the McElroy study, Zeljkovic et al. imaged type-A oxygens as bright features in $\mathrm{d} I / \mathrm{d} V$ images at around $-1.5 \mathrm{~V}$ bias, ${ }^{71}$ which also turned out to correlate with the areas of large PG (Fig. 10f). However, this study also revealed another a third set of oxygenrelated defects in $\mathrm{d} I / \mathrm{d} V$ images at $+1 \mathrm{~V}$ bias, identified as apical $O$ vacancies (AOVs). Positions of the AOVs were found to correlate well with the areas of large PG (Fig. 10f), finally an expected trend, as each AOV locally removes 2 holes from the system and should increase the PG both locally and globally (Fig. 1a). Furthermore, AOVs are found to lie primarily at the peaks of the "checkerboard" charge modulation (an order possibly associated with the PG state ${ }^{94,98-100,104}$ ) (Fig. 10g). ${ }^{71}$ Therefore, Zeljkovic et al. concluded that AOVs in underdoped Bi-2212 samples restore localized electrons to the $\mathrm{CuO}_{2}$ plane, whereas both types of interstitial oxygens contribute primarily delocalized holes and are likely a secondary factor in any spectral inhomogeneity. ${ }^{71}$
Since Bi-2212 crystals are difficult to synthesize in the perfect stoichiometric form, ${ }^{105}$ extra $\mathrm{Bi}$ is commonly used to facilitate the growth process. Most crystals contain $\sim 5-10 \%$ excess $\mathrm{Bi}$ substituted at the $\mathrm{Sr}$ site. ${ }^{105}$ Because the $\mathrm{Sr}$ site is located next to the apical $\mathrm{O}$ site (which is directly above $\mathrm{Cu}$, see Fig. 10a), it might be expected that a substitution at this site would have a strong effect on the electronic properties of the crucial $\mathrm{CuO}_{2}$ plane. Kinoda et al. imaged Sr site defects as a set of bright features in STM topographs of Pb-doped Bi-2212 at biases greater than $+1.4 \mathrm{~V}$. The $\mathrm{d} I / \mathrm{d} V$ spectrum on top of a $\mathrm{Sr}$ site defect shows a resonance peak at $+1.7 \mathrm{~V}$ (Fig. 10e). Although no quantitative analysis was presented, ${ }^{103}$ we have analyzed the published data to obtain a small positive correlation between the Sr site defect locations and the PG (Fig. 10f). Hence, although not the strongest correlation, Sr site defects also seem to be correlated with the observed PG inhomogeneity.

An additional complication in many Bi-based cuprates is the structural "supermodulation" (SM), a 25 A incommensurate sinusoidal modulation that pervades the bulk of the material. It is seen in STM topographs with additional quasiperiodic distortions of atoms in the shape of a dollar-sign ("\$") at the modulation crests (Fig. 10b). Pb dopants substituted at the $\mathrm{Bi}$ sites can be used to partially or completely suppress this superstructure. ${ }^{106}$ Kinoda et al. studied heavily-overdoped Bi-2212 crystals with $\mathrm{Pb}$ substitutions ${ }^{103}$ and claimed to observe no 

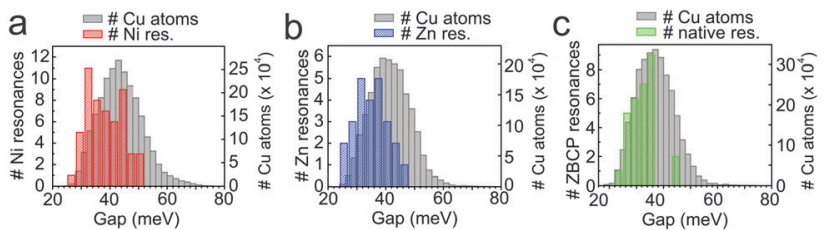

Fig. 11 Pseudogap and Cu site defect histograms in Bi-2212. (a-c) Histograms of pseudogap at the defect sites are shown in blue ( $\mathrm{Zn}$ substitutions), red (Ni substitutions), and green (native defects). Histograms of pseudogap spectral surveys for the whole sample are shown in gray. No Cu site defects are observed in the regions with pseudogap larger than $50 \mathrm{meV}^{62,92}$

correlation between the $\mathrm{Pb}$ dopant positions and the magnitude of the spectral gap (Fig. 10e), therefore ruling out these dopants as the cause or the pinning sites of the nanoscale heterogeneity. The lack of correlation between $\mathrm{Pb}$ dopants and the spectral gap was consequently confirmed in $\mathrm{Pb}$-doped Bi-2201 crystals. ${ }^{107}$ Both experiments seem to agree that although $\mathrm{Pb}$ dopants add carriers and alter structure, their effect on the electronic properties is not localized.

The final source of chemical disorder in Bi-2212 is $\mathrm{Cu}$ site defects $\left(\mathrm{Zn}^{40}\right.$ and $\mathrm{Ni}^{43}$ substitutions and intrinsic defects thought to be vacancies ${ }^{41}$ ), which have a tendency to appear in the areas of small spectral gap ( $<50 \mathrm{meV}$ ) (Fig. 11). However, since spectral gap inhomogeneity is observed in the absence of $\mathrm{Zn}$ or Ni doping (or intrinsic $\mathrm{Cu}$ site defects which we observed only rarely in our extensive studies of $\mathrm{Bi}-2212$ ), we rule these out as the cause of the spectral gap variation. Nevertheless, due to the correlation of their distributions with the areas of the small spectral gap, it seems plausible that the electronic inhomogeneity could be pinned by these impurities.

Na-CCOC. Both type $\mathrm{I}^{108}$ and type $\mathrm{II}^{109}$ inhomogeneity have also been observed in another hole-doped cuprate, Na-CCOC. In contrast to $\mathrm{Bi}-2212$, the parent compound $\mathrm{Ca}_{2} \mathrm{CuO}_{2} \mathrm{Cl}_{2}$ (CCOC) can more readily be grown and is therefore an ideal material in which to investigate the evolution with doping of the electronic properties from the antiferromagnetic Mott insulator state to the superconducting state (Fig. 1a). However, due to the insulating nature of the CCOC parent compound (Fig. 12a) at $4 \mathrm{~K}$ where most STM studies on these compounds are carried out, the material has been difficult to study using STM. Recently, Ye et al. have made a breakthrough by studying parent CCOC at $77 \mathrm{~K}$. The topographs of stoichiometric CCOC are reported to be homogeneous (Fig. 12c), ${ }^{110}$ in contrast to those of lightly-doped Na-CCOC both in non-superconducting and barely superconducting states, shown in Fig. $12 \mathrm{~d}-\mathrm{f} .{ }^{108} \mathrm{Ye}$ et al. imaged both electron donors ( $\mathrm{Cl}$ vacancies) and hole donors (Ca site defects, thought to be vacancies or Na substitutions). In both cases, the defects locally moved spectral weight from the upper Hubbard band into the charge transfer gap, while leaving the position of the Fermi level unchanged, and the Fermi level density of states zero (Fig. 12b). The in-gap state has a broad energy distribution and is strongly localized in space, suggesting that in this near-parent compound the doped charge does not behave like a coherent quasiparticle, and that the impurity potential is poorly screened, presumably owing to

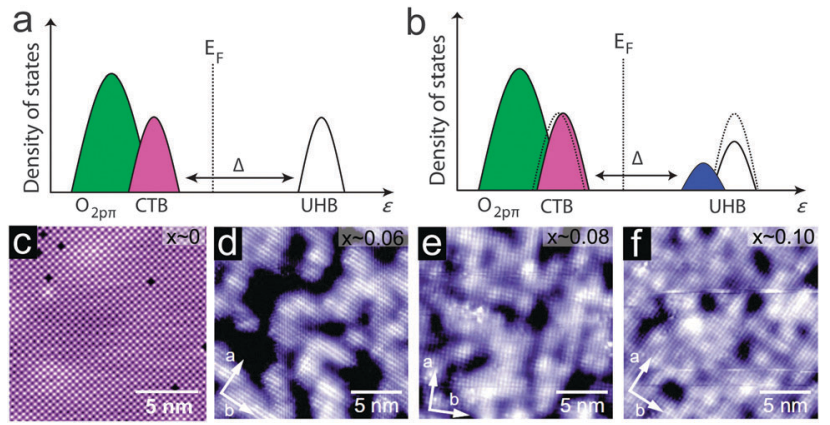

Fig. 12 Band structure and electronic inhomogeneity in $\mathrm{Na}_{x} \mathrm{Ca}_{2-x} \mathrm{CuO}_{2} \mathrm{Cl}_{2}$. (a) Schematic band structure of the pristine Mott insulator showing the upper Hubbard band (UHB, unfilled), the charge transfer band (CTB, pink) and the non-bonding oxygen $2 \mathrm{p} \pi$ band (green). (b) Schematic electronic structure at a $\mathrm{Cl}$ vacancy defect center: an in-gap state (blue) emerges near the edge of UHB owing to spectral weight transfer from the latter. The CTB is slightly shifted and the local gap amplitude is significantly reduced. (c-f) Topographs of the parent compound with $x=0$ (c), ${ }^{110}$ and Na-CCOC with $x=0.06\left(T_{c}=0 \mathrm{~K}\right)(\mathrm{d}), x=0.08$ $\left(T_{\mathrm{c}}=0 \mathrm{~K}\right)(\mathrm{e})$, and $x=0.10\left(T_{\mathrm{c}}=13 \mathrm{~K}\right)(\mathrm{f}) .{ }^{108}$ Setup conditions are $(\mathrm{c})-3.5 \mathrm{~V}, 10 \mathrm{pA}$, $77 \mathrm{~K}$; (d) $300 \mathrm{mV}, 100 \mathrm{pA}, 5 \mathrm{~K}$; and (e-f) $200 \mathrm{mV}, 100 \mathrm{pA}, 4.7 \mathrm{~K}$.

the strong correlation effect. Further experiments bridging the unstudied doping range between this parent compound (Fig. 12c) and the 6\% doped compound (Fig. 12d), simultaneously imaging the dopant atoms and the growing patches of electronic inhomogeneity, may shed additional light on the interplay between strong correlations and impurities. However, to definitively answer the question whether electronic inhomogeneity would arise from strong correlations alone in the absence of chemical inhomogeneity, one must image a gate-doped stoichiometric parent compound. Ye's recent work has paved the way, demonstrating the imageability of near-stoichiometric CCOC at the relatively low temperature of $77 \mathrm{~K}$ where thermal broadening $\sim 4 k_{\mathrm{B}} T=27 \mathrm{meV}$ is still small compared to the reported energy scales of the inhomogeneity. Gate doping of two other cuprates, $\mathrm{YBCO}^{111,112}$ and $\mathrm{La}_{2-x} \mathrm{Sr}_{x} \mathrm{CuO}_{4},{ }^{113,114}$ from the Mott insulator to the superconducting state has recently been demonstrated, but further work is needed to accomplish this feat in a geometry amenable to STM.

YBCO. YBCO lacks a natural cleavage plane, but cryogenicallycleaved surfaces usually reveal either the BaO plane ${ }^{115-118}$ or CuO chain plane. ${ }^{115,117-121}$ Although these surfaces may be imaged with atomic resolution, neither show a superconducting gap. In contrast, as-grown YBCO surfaces show a gap plausibly identified as the superconducting gap, but neither atomic resolution nor type I inhomogeneity have been observed. ${ }^{122}$

The cleaved $\mathrm{CuO}$ chain surface exhibits a complicated onedimensional modulation (Fig. 13a) initially attributed to a CDW state. ${ }^{119,120,123}$ Derro et al. performed additional experiments on the $\mathrm{CuO}$ chain surface to reveal a set of atomic-scale resonances (likely to be $\mathrm{O}$ vacancies) exclusively present at sub-gap energies below $25 \mathrm{meV} .{ }^{124}$ Different sets of resonances "light up" at different energies in $\mathrm{d} I / \mathrm{d} V$ images (Fig. 13b-d). A typical $\mathrm{d} I / \mathrm{d} V$ spectrum on top of one of these is shown in Fig. 13e, and exhibits two main resonances and their "shadows" at corresponding opposite biases. Derro et al. suggested that these 

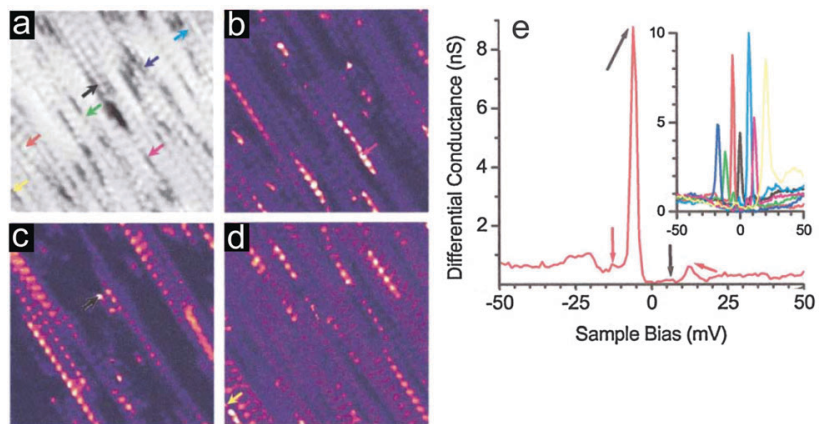

Fig. 13 Spectroscopy of CuO chain plane in YBCO. Simultaneously obtained (a) $25 \mathrm{~nm}$ square topograph, and (b-d) $\mathrm{d} / / \mathrm{d} V$ maps obtained at $0 \mathrm{mV}, 6 \mathrm{mV}$ and $12 \mathrm{mV}$ respectively (200 pA, $-200 \mathrm{mV}, 4.2 \mathrm{~K}$ ). (e) Typical $\mathrm{d} / / \mathrm{d} V$ spectrum acquired at the position indicated by the red arrow in (a). Other colored arrows in (a) correspond to different positions on the sample where the $d / / d V$ spectra shown in the inset of (e) were acquired. ${ }^{124}$

resonances might be a consequence of superconducting quasiparticle scattering rather than an indication of a CDW state as previously reported ${ }^{120,123}$ for two reasons. First, the wavelength of these resonances in real space disperses by more than $10 \%$ within $50 \mathrm{meV}$ away from the Fermi level, whereas a CDW typically has a fixed wavelength determined by the nesting vectors on the Fermi surface. Second, similar resonances have been observed in Bi-2212 (ref. 38-40 and 43) as a consequence of atomic defects. Morr and Balatsky proposed that one-dimensional scattering resonances can be observed in the chain plane of YBCO if the $\mathrm{CuO}$ plane is also superconducting due to its proximity to the $\mathrm{CuO}_{2}$ plane. ${ }^{125}$ However, this explanation seems unlikely as no superconducting gap on the surface was conclusively presented by Derro et al., and cleaved YBCO crystals seem to suffer from surface overdoping all the way out of the superconducting state. ${ }^{126,127}$

In summary, patchy (type I) and periodic (type II) electronic inhomogeneities are both observed across several cuprate compounds. There exists some experimental evidence that both types of inhomogeneity are pinned by specific defects, but there is no conclusive evidence that chemical inhomogeneity is solely responsible for either type of electronic inhomogeneity. No studies have uncovered ordered dopants which could plausibly cause type II inhomogeneity. In Bi-2212, recent studies suggest that AOVs act as the strongest pinning sites of large PG "patches" (type I inhomogeneity) and peaks of "checkerboard" charge modulation (type II inhomogeneity). ${ }^{71}$ However, AOVs occur only in underdoped Bi-2212 and even in those samples, they are not seen in all large PG patches (e.g. the region in Fig. 10c denoted by an arrow). Cu site defects have also been shown to pin the areas of small spectral gap, ${ }^{62}$ but likewise they do not occur in all Bi-2212 samples studied, nor are they seen in all small gap areas. Sr site defects exhibit some correlation with the areas of large PG. Other impurities, such as Pb dopants and some interstitial oxygens, seem to exhibit little correlation with the PG. Yet no study to date has imaged all of the aforementioned dopants in the same field of view, and it would be interesting to see the extent to which the spectral gap maps (see Fig. 10c for an example) can be reconstructed based on the knowledge of all dopant positions. ${ }^{128}$ In CCOC, electronic inhomogeneity might be a direct consequence of $\mathrm{Na}$ dopant disorder, but further work is required to conclusively show this. In YBCO, one-dimensional resonances observed in the $\mathrm{CuO}$ chain layer are attributed to $\mathrm{O}$ vacancies, ${ }^{124}$ but due to the surface overdoping of YBCO, ${ }^{126}$ these one-dimensional resonances may not be representative of the bulk. The definitive answer to the question of whether chemical inhomogeneity causes or merely pins type I electronic inhomogeneity will likely come only from studies of gate-doped stoichiometric parent cuprates.

\section{Chemical disorder and $T_{\mathrm{c}}$}

An important metric of superconducting utility is its $T_{\mathrm{c}}$, the temperature below which the resistance of the material drops to zero. Within both cuprates and Fe-SCs, the bulk compounds with highest $T_{\mathrm{c}}$ are all non-stoichiometric, with maximum ambient pressure $T_{\mathrm{c}}$ of $\sim 135 \mathrm{~K}$ in $\mathrm{HgBa}_{2} \mathrm{Ca}_{2} \mathrm{Cu}_{3} \mathrm{O}_{x}{ }^{129}$ and $\sim 57 \mathrm{~K}$ in $\mathrm{Sm}_{1-x} \mathrm{La}_{x} \mathrm{O}_{1-y} \mathrm{~F}_{y}$ FeAs. ${ }^{16}$ Nominally stoichiometric superconductors tend to have lower $T_{\mathrm{c}}$ (such as $81 \mathrm{~K}$ in $\mathrm{YBa}_{2} \mathrm{Cu}_{4} \mathrm{O}_{8}$ (ref. 130) and $18 \mathrm{~K} \mathrm{LiFeAs}{ }^{131}$ ). These comparisons beg the question whether chemical disorder itself may play a role in enhancing $T_{\mathrm{c}}$.

To address the effect of dopant disorder on $T_{\mathrm{c}}$ in cuprates, Eisaki et al. thoroughly investigated cation substitutions in Bi-2201, and discovered that partial replacement of $\mathrm{Sr}^{2+}$ by rare-earth ions resulted in a monotonic decrease of $T_{\mathrm{c}}$ with increasing ionic radius mismatch. ${ }^{105} \mathrm{~A}$ second test in $\mathrm{Bi}-2212$ reinforced this point: by minimizing the concentration of $\mathrm{Bi}$ substitutions at the $\mathrm{Sr}$ site, $T_{\mathrm{c}}$ was increased from $82.4 \mathrm{~K}$ for $\sim 20 \%$ substitutions to $94.0 \mathrm{~K}$ for the pure sample with $\mathrm{Bi}: \mathrm{Sr}$ ratio closest to $1: 1$. Substitution of $8 \% \mathrm{Y}$ atoms at the Ca site allowed even better Sr site stoichiometry, increasing $T_{\mathrm{c}}$ still further to $96 \mathrm{~K}$, and emphasizing that not all types of disorder are equally harmful.

Since disorder in the cuprate superconducting $\mathrm{CuO}_{2}$ planes depresses $T_{\mathrm{c}}{ }^{132}$ it was surprising that some Fe-SCs arise by doping directly into the crucial FeAs plane of a non-superconducting parent compound. For example, $T_{\mathrm{c}} \sim 25 \mathrm{~K}$ in $\mathrm{Ba}\left(\mathrm{Fe}_{1-x} \mathrm{Co}_{x}\right)_{2} \mathrm{As}_{2},{ }^{133}$ a member of the electron-doped $A\left(\mathrm{Fe}_{1-x} \mathrm{M}_{x}\right)_{2} \mathrm{As}_{2}$ (A122) family, where $\mathrm{M}=\mathrm{Co}, \mathrm{Ni}$, etc. It was pointed out that $T_{\mathrm{c}}$ in electron-doped $A 122$ Fe-SCs could potentially be even higher, comparable to the $T_{\text {c,max }}=38 \mathrm{~K}$ found in hole-doped $A 122$ materials, if the damaging dopant disorder were taken out of the FeAs layer and moved into the buffer layer between the two FeAs planes. Indeed, this was found to be the case in the rare-earth-doped Ca122 family, ${ }^{134,135}$ with $T_{\text {c }}$ reaching $49 \mathrm{~K}$ in $\operatorname{Pr}_{x} \mathrm{Ca}_{1-x} \mathrm{Fe}_{2} \mathrm{As}_{2}$ (Pr-Ca122). However, the high $T_{\mathrm{c}}$ appeared in only $\sim 10 \%$ of the volume, while the bulk of the material showed $T_{\mathrm{c}} \sim 10-20 \mathrm{~K}$. Extensive tests have so far failed to determine the origin of this mysterious low volume fraction high- $T_{\mathrm{c}}$ phase, as they appear to have ruled out surface superconductivity, contaminant phases, and strain mechanics, ${ }^{135}$ leaving dopant clustering as one of the few remaining hypotheses. Zeljkovic et al. recently addressed this issue by performing realspace imaging of Pr dopants in Pr-Ca122. ${ }^{136}$ The Pr dopants appear more evenly distributed than would be expected from a completely random distribution (possibly due to like charge 
repulsion between $\operatorname{Pr}^{3+}$ ions); thus Pr clustering is an unlikely cause of the low volume fraction high- $T_{\mathrm{c}}$ phase in this material.

Even though dopant distribution did not explain the mystery of the high- $T_{\mathrm{c}}$ phase in Pr-Ca122, several other studies have emphasized the importance of specific arrangements of dopants, for creating better superconductors with higher $T_{\mathrm{c}}$. By coupling layers of $\mathrm{La}_{2} \mathrm{CuO}_{4+\delta}$ to metallic $\mathrm{La}_{1.55} \mathrm{Sr}_{0.45} \mathrm{CuO}_{4}$, neither of which have $T_{\mathrm{c}}$ greater than $48 \mathrm{~K}$ in isolation, Gozar et al. increased the $T_{\mathrm{c}}$ up to $\sim 51 \mathrm{~K} .{ }^{137}$ Goren and Altman predicted $a \sim 15 \%$ increase in $T_{\mathrm{c}}$ from alternate dopant arrangements within the $\mathrm{CuO}_{2}$ planes of $\mathrm{Bi}-2212 .^{138}$ It was recently reported that a single layer of FeSe on top of $\mathrm{SrTiO}_{3}$ substrate has $T_{\mathrm{c}} \sim 65 \mathrm{~K}^{18}$ which is much higher than the bulk $T_{\mathrm{c}}$ of FeSe. The efforts to synthesize materials with higher $T_{\mathrm{c}}$ are ongoing, and these experiments demonstrate the significance of inhomogeneity in achieving that feat.

\section{Chemical disorder and vortex pinning}

Another metric of superconductor performance is its behavior in magnetic field. When a magnetic field is applied to a type I superconductor, magnetic flux is expelled to a thin layer on the surface. In contrast, in type II superconductors, magnetic flux penetrates as quantized flux tubes called vortices. Because they avoid the high energy cost of complete flux expulsion, type II superconductors tend to have higher upper critical temperatures and fields. Their utility is typically limited, however, by the unwanted motion of vortices, driven by the Lorentz force $F=J \times B$ perpendicular to a flowing supercurrent $J$. Because the Cooper pairs are broken at the vortex core, the motion of vortex cores is dissipative. A vortex may be pinned by co-locating its normal state core with a crystal defect which also suppresses superconductivity, to avoid paying the energy cost of lost superconductivity twice. The critical current $J_{\mathrm{c}}$ is therefore determined by the balance between the Lorentz force and the defect pinning force. Thus tremendous research effort spanning many decades has been devoted to fine-tuning chemical impurities to strengthen vortex pinning without significantly degrading other superconducting metrics. ${ }^{37,139}$ Most of this effort is based on tweaking the chemical structure and measuring the bulk critical current, blind to the individual interactions between vortices and specific pinning sites. Interpretation of these bulk experiments can be challenging, particularly in high- $T_{\mathrm{c}}$ materials which may already host several forms of inhomogeneity even before the introduction of potential new pinning sites.

The most commercialized high- $T_{\mathrm{c}}$ material for the last decade has been YBCO. ${ }^{140}$ STM and magnetic force microscopy (MFM) have been applied to understand vortex pinning in pristine single crystal YBCO. Maggio-Aprile et al. imaged an oblique vortex lattice with no apparent long-range order in YBCO. ${ }^{141}$ The lattice axes were seen to rotate $90^{\circ}$ at a twin boundary, as would be expected if the pinning were related to the $\mathrm{CuO}$ chain planes. ${ }^{142}$ Although no atomic resolution was achieved in these studies (and therefore no atomic defects were imaged), previous transport experiments suggested the importance of oxygen vacancies in pinning the magnetic flux lines. ${ }^{143}$ Several STM studies observed $\mathrm{O}$ vacancies in the $\mathrm{CuO}$ chain layer, ${ }^{118,120}$ which were reported to form anisotropic clusters typically 2-4 $\mathrm{nm}$ in size, ${ }^{118}$ thus providing a real-space confirmation of oxygen vacancy clustering in this material. Subsequently, MFM confirmed the importance of oxygen vacancy clusters in vortex flux pinning, estimating cluster size to be $\sim 10$ vacancies. ${ }^{144}$ For commercial applications, pristine single crystal YBCO growth is not viable, but cheaper tape growth must introduce sufficient pinners while minimizing grain boundaries. Even grain boundaries with as small as $4^{\circ}$ misalignment act as weak links, exponentially degrading the critical current with increasing angle. ${ }^{145}$ Recently, bulk transport measurements have shown that Zr doping improves critical current in field, ${ }^{140}$ but microscopic mechanisms have not been investigated at the single vortex level.

STM has been used in recent years to directly image individual vortices and pinning sites in the Fe-SCs, ${ }^{146}$ where low anisotropy, high upper critical field, and strong native vortex pinning motivate studies to evaluate whether Fe-SCs may eventually be more profitable for applications. ${ }^{147}$ Although STM images only the surface, where the vortex lines emerge from the bulk of the material, it can nonetheless give valuable information about the vortex arrangement in the interior. From the relationship between vortex tops and surface defects, the electronic anisotropy can be inferred. A relatively isotropic material will have a high energy cost for a vortex to bend, so the vortices will have little chance to co-locate with all the impurities in any specific chemical layer, but instead will bend only slightly to maximize the overlap with impurities throughout the bulk of the crystal. However, a highly anisotropic material can be easily identified by the tendency of vortices to pin on impurities in the surface layer, thus signifying their ease of bending or even their separation into individual "pancake" vortices. ${ }^{148}$ For example, $\mathrm{Ba}\left(\mathrm{Fe}_{1-x} \mathrm{Co}_{x}\right)_{2} \mathrm{As}_{2}$ is found to be a relatively isotropic superconductor, as the vortex tops are uncorrelated with a set of surface defects which clearly independently destroy local superconductivity. ${ }^{149}$ In contrast, Bi-2212 is found to be a highly anisotropic superconductor, in which the majority of vortex tops appear pinned by surface $\mathrm{Zn}$ impurities. ${ }^{150}$

From the vortex arrangement alone, the relative strength of the pinning force and the inter-vortex interactions can be inferred. For example, in $\mathrm{K}_{x} \mathrm{Ba}_{1-x} \mathrm{Fe}_{2} \mathrm{As}_{2}$, inter-vortex interactions apparently dominate, and the vortices form a hexagonal lattice (Fig. 14a). ${ }^{151}$ However, in both $\mathrm{Ba}\left(\mathrm{Fe}_{1-x} \mathrm{Co}_{x}\right)_{2} \mathrm{As}_{2}$ (Fig. 14b) ${ }^{149}$ and $\mathrm{K}_{x} \mathrm{Sr}_{1-x} \mathrm{Fe}_{2} \mathrm{As}_{2}$ (Fig. 14c), ${ }^{152}$ the vortices form a disordered lattice, indicating stronger pinning. Song et al. proposed that this contrast stems from differences in dopant size mismatch: larger size mismatch leads to dopant clustering to relax the strain, which in turn leads to more significant electronic inhomogeneity and stronger vortex pinning. ${ }^{152}$ Electronic inhomogeneity arising from dopant clustering is supported also in the $\mathrm{FeSe}_{1-x} \mathrm{Te}_{x}$ system. In $\mathrm{FeTe}_{0.55} \mathrm{Se}_{0.45}$, He et al. used statistical analysis of dopant positions to reveal dopant clustering of $\sim 1 \mathrm{~nm}^{2}$ size, ${ }^{153}$ which likely pin the disordered vortex structure. $^{72}$ (Even in nominally stoichiometric LiFeAs (Fig. 14d), the vortex structure is disordered possibly due to intrinsic defects similar to the ones shown in Fig. $5 \mathrm{e}-\mathrm{h} .{ }^{53}$ ) 

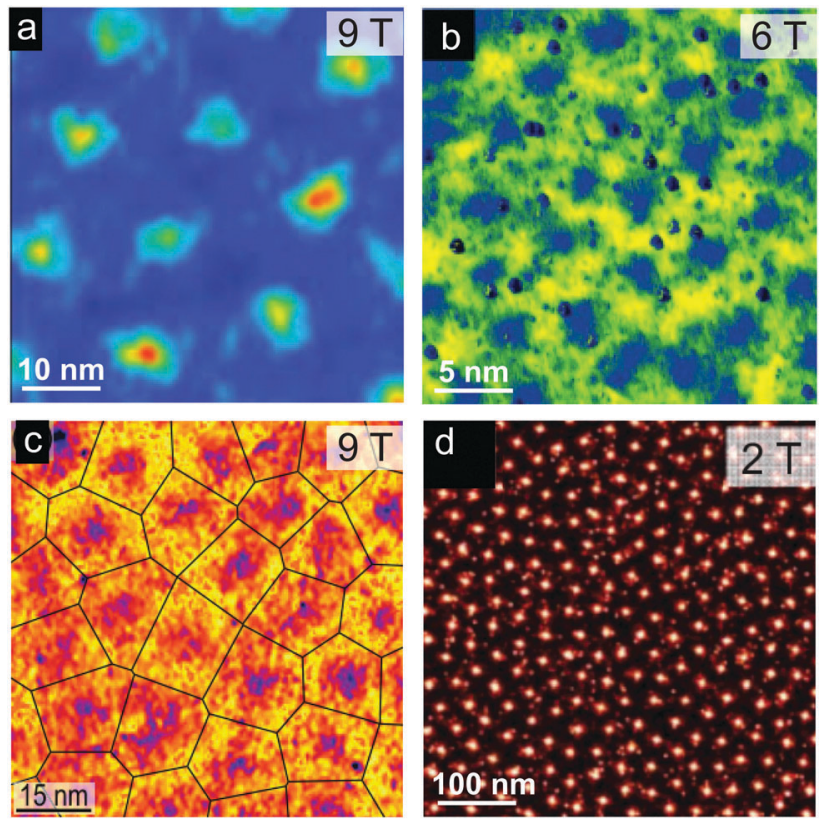

Fig. 14 Vortex pinning in high- $T_{c}$ superconductors. (a) Zero-bias conductance map recorded at $9 \mathrm{~T}$ magnetic field showing an ordered vortex lattice in $\mathrm{Ba}_{0.6} \mathrm{~K}_{0.4} \mathrm{Fe}_{2} \mathrm{As}_{2}(100 \mathrm{mV}, 200 \mathrm{pA}) .{ }^{151}$ (b) $\mathrm{d} / / \mathrm{dV}(-5 \mathrm{mV})$ image of $\mathrm{Ba}\left(\mathrm{Fe}_{1-x} \mathrm{CO}_{x}\right)_{2} \mathrm{As}_{2}$ showing a disordered vortex lattice not pinned to surface impurities at $6 \mathrm{~T}$ magnetic field. ${ }^{149}$ (c) $\mathrm{A} d / / \mathrm{dV}(-5 \mathrm{mV})$ image showing vortices at $9 \mathrm{~T}$ in $\mathrm{K}_{x} \mathrm{Sr}_{1-x} \mathrm{Fe}_{2} \mathrm{As}_{2}{ }^{152}$ (d) Zero-bias-conductance image showing a disordered vortex structure in LiFeAs at $2 \mathrm{~T}$ magnetic field $(20 \mathrm{mV}, 100 \mathrm{pA}, 1.5 \mathrm{~K}) .^{53}$

In summary, dopant disorder strongly influences both $T_{\mathrm{c}}$ and $J_{\mathrm{c}}$ in high- $T_{\mathrm{c}}$ superconductors, but in opposing manners minimizing defects at specific lattice sites can raise $T_{\mathrm{c}}$, but the same reduced disorder is less effective at pinning magnetic flux lines, thus lowering $J_{c}$. These conflicting effects complicate the efforts to synthesize new superconductors with simultaneously enhanced metrics $T_{\mathrm{c}}$ and $J_{\mathrm{c}}$, but a possible avenue of investigation in layered materials could involve moving dopants further away from the superconducting plane while maintaining the same degree of disorder, as in the case of the Ca-doped Bi-2212 (ref. 105) and rare-earth-doped Fe-SCs. ${ }^{134,135}$

\section{Conclusions and future directions}

Over the past several decades, scanning tunneling microscopy has made tremendous contributions to the understanding of unconventional superconductors. In contrast to bulk-probe techniques that measure electronic properties averaged over large areas of a material, STM has a unique capability to simultaneously access structural and electronic properties at the atomic scale. STM is therefore the ideal tool for studies of chemical disorder and its effects.

In this Perspective, we have addressed several issues. First, we reviewed how STM can constrain the pairing symmetry in unconventional superconductors by using spectroscopic measurements at specific atomic defect sites. Furthermore, impurities can be intentionally introduced into unconventional superconductors as scattering sites for QPI imaging, which reveals the underlying band structure and symmetry breaking in momentum space. Second, we have shown that electronic inhomogeneity in cuprate superconductors is likely pinned by specific chemical defects, but there is little experimental evidence to determine whether the electronic inhomogeneity would arise spontaneously in the absence of such defects. Finally, we discussed how chemical disorder can be used to enhance desired superconducting properties such as $T_{\mathrm{c}}$ or $J_{\mathrm{c}}$.

There are still many unexplored aspects of chemical and electronic inhomogeneity in unconventional superconductors. First, STM is constrained to measuring atomically flat and clean surfaces, which are usually achieved by cleaving single crystals. Although there has been considerable progress in growth and study of Fe-SCs using combined MBE-STM systems, many unconventional superconductors, such as $\mathrm{YBa}_{2} \mathrm{Cu}_{3} \mathrm{O}_{7-x}$, $\mathrm{La}_{2-x} \mathrm{Sr}_{x} \mathrm{CuO}_{4}$, or $\mathrm{Ba}_{1-x} \mathrm{~K}_{x} \mathrm{BiO}_{3},{ }^{127,154}$ cannot be cleaved to expose atomically flat surfaces with electronic states clearly representative of the bulk. Second, spin-polarized STM has been very successful in spin-resolved imaging of systems of magnetic elements ${ }^{155}$ yet SP-STM has not yet been successfully applied to unconventional superconductors. STM will surely continue to make significant contributions towards understanding the role of chemical disorder in shaping the electronic properties of superconductors. This improved understanding will enable enhancements to the properties of today's superconductors, as well as predictions to discover and synthesize new and better superconductors in the future.

We thank Can-Li Song and Dennis Huang for helpful conversations. This work was supported by the Air Force Office of Scientific Research under grant FA9550-06-1-0531, and the U.S. National Science Foundation under grant DMR-0847433.

\section{References}

1 D. van Delft and P. Kes, Phys. Today, 2010, 63, 38.

2 W. Meissner and R. Ochsenfeld, Die Naturwiss., 1933, 21, 787.

3 J. Bardeen, L. N. Cooper and J. R. Schrieffer, Phys. Rev., 1957, 108, 1175.

4 W. McMillan, Phys. Rev., 1968, 167, 331.

5 J. G. Bednorz and K. A. Muller, Z. Phys. B: Condens. Matter, 1986, 64, 189.

6 M. Wu, J. Ashburn, C. Torng, P. Hor, R. Meng, L. Gao, Z. Huang, Y. Wang and C. Chu, Phys. Rev. Lett., 1987, 58, 908.

7 P. A. Lee, N. Nagaosa and X.-G. Wen, Rev. Mod. Phys., 2006, 78, 17.

8 P. Phillips, Rev. Mod. Phys., 2010, 82, 1719.

9 S. Hüfner, M. A. Hossain, A. Damascelli and G. A. Sawatzky, Rep. Prog. Phys., 2008, 71, 062501.

10 V. J. Emery and S. A. Kivelson, Nature, 1995, 374, 434.

11 Y. Kamihara, T. Watanabe, M. Hirano and H. Hosono, J. Am. Chem. Soc., 2008, 130, 3296.

12 D. N. Basov and A. V. Chubukov, Nat. Phys., 2011, 7, 272.

13 D. C. Johnston, Adv. Phys., 2010, 59, 803.

14 G. Stewart, Rev. Mod. Phys., 2011, 83, 1589. 
15 K. Deguchi, Y. Mizuguchi, Y. Kawasaki, T. Ozaki, S. Tsuda, T. Yamaguchi and Y. Takano, Supercond. Sci. Technol., 2011, 24, 055008.

16 Z. Wei, H. Li, W. Hong, Z. Lv, H. Wu, X. Guo and K. Ruan, J. Supercond. Novel Magn., 2008, 21, 213.

17 C. Wang, L. Li, S. Chi, Z. Zhu, Z. Ren, Y. Li, Y. Wang, X. Lin, Y. Luo, S. Jiang, X. Xu, G. Cao and Z. Xu, Europhys. Lett., 2008, 83, 67006 .

18 S. He, J. He, W. Zhang, L. Zhao, D. Liu, X. Liu, D. Mou, Y.-B. Ou, Q.-Y. Wang, Z. Li, L. Wang, Y. Peng, Y. Liu, C. Chen, L. Yu, G. Liu, X. Dong, J. Zhang, C. Chen, Z. Xu, X. Chen, X. Ma, Q. Xue, X. J. Zhou, arXiv:1207.6823.

19 C. Pfleiderer, Rev. Mod. Phys., 2009, 81, 1551.

20 C. C. Tsuei and J. R. Kirtley, Rev. Mod. Phys., 2000, 72, 969.

21 P. J. Hirschfeld, M. M. Korshunov and I. I. Mazin, Rep. Prog. Phys., 2011, 74, 124508.

22 G. Binnig, H. Rohrer, C. Gerber and E. Weibel, Phys. Rev. Lett., 1982, 49, 57.

23 J. Tersoff and D. R. Hamann, Phys. Rev. B: Condens. Matter Mater. Phys., 1985, 31, 805.

24 A. V. Balatsky, I. Vekhter and J.-X. Zhu, Rev. Mod. Phys., 2006, 78, 373.

25 J. Byers, M. Flatté and D. Scalapino, Phys. Rev. Lett., 1993, 71, 3363.

26 M. Salkola, A. Balatsky and D. Scalapino, Phys. Rev. Lett., 1996, 77, 1841.

27 M. I. Salkola, A. V. Balatsky and J. R. Schrieffer, Phys. Rev. B: Condens. Matter Mater. Phys., 1997, 55, 12648.

28 Y. Bang, H.-Y. Choi and H. Won, Phys. Rev. B: Condens. Matter Mater. Phys., 2009, 79, 054529.

29 D. Zhang, Phys. Rev. Lett., 2009, 103, 186402.

30 W.-F. Tsai, Y.-Y. Zhang, C. Fang and J. Hu, Phys. Rev. B: Condens. Matter Mater. Phys., 2009, 80, 064513.

31 M. Matsumoto, M. Koga and H. Kusunose, J. Phys. Soc. Jpn., 2009, 78, 084718.

32 T. Ng and Y. Avishai, Phys. Rev. B: Condens. Matter Mater. Phys., 2009, 80, 104504.

33 T. Kariyado and M. Ogata, J. Phys. Soc. Jpn., 2010, 79, 083704.

34 R. Beaird, I. Vekhter and J.-X. Zhu, Phys. Rev. B: Condens. Matter Mater. Phys., 2012, 86, 140507.

35 H. Maeda, Y. Tanaka, M. Fukutomi and T. Asano, Jpn. J. Appl. Phys., 1988, 27, L209.

36 M. A. Subramanian, C. C. Torardi, J. C. Calabrese, J. Gopalakrishnan, K. J. Morrissey, T. R. Askew, R. B. Flippen, U. Chowdhry and A. W. Sleight, Science, 1988, 239, 1015.

37 D. Larbalestier, A. Gurevich, D. M. Feldmann and A. Polyanskii, Nature, 2001, 414, 368.

38 E. W. Hudson, S.-H. Pan, A. K. Gupta, K.-W. Ng and J. C. Davis, Science, 1999, 285, 88.

39 A. Yazdani, C. Howald, C. Lutz, A. Kapitulnik and D. Eigler, Phys. Rev. Lett., 1999, 83, 176.

40 S.-H. Pan, E. W. Hudson, K. M. Lang, H. Eisaki, S. Uchida and J. C. Davis, Nature, 2000, 403, 746.

41 E. Hudson, Phys. B, 2003, 329-333, 1365.
42 K. Chatterjee, M. C. Boyer, W. D. Wise, T. Kondo, T. Takeuchi, H. Ikuta and E. W. Hudson, Nat. Phys., 2008, 4, 108.

43 E. W. Hudson, K. M. Lang, V. Madhavan, S.-H. Pan, H. Eisaki, S. Uchida and J. C. Davis, Nature, 2001, 411, 920. 44 D. Van Harlingen, Rev. Mod. Phys., 1995, 67, 515.

45 C.-L. Song, Y.-L. Wang, P. Cheng, Y.-P. Jiang, W. Li, T. Zhang, Z. Li, K. He, L. Wang, J.-F. Jia, H.-H. Hung, C. Wu, X. Ma, X. Chen and Q.-K. Xue, Science, 2011, 332, 1410.

46 S. Grothe, S. Chi, P. Dosanjh, R. Liang, W. Hardy, S. Burke, D. Bonn and Y. Pennec, Phys. Rev. B: Condens. Matter Mater. Phys., 2012, 86, 174503.

47 W. Li, H. Ding, P. Deng, K. Chang, C. Song, K. He, L. Wang, X. Ma, J.-P. Hu, X. Chen and Q.-K. Xue, Nat. Phys., 2011, 8, 126.

48 W. Lv, J. Wu and P. Phillips, Phys. Rev. B: Condens. Matter Mater. Phys., 2009, 80, 224506.

49 C.-C. Lee, W.-G. Yin and W. Ku, Phys. Rev. Lett., 2009, 103, 267001.

50 C.-L. Song, Y.-L. Wang, Y.-p. Jiang, L. Wang, K. He, X. Chen, J. Hoffman, X.-c. Ma and Q.-k. Xue, Phys. Rev. Lett., 2012, 109, 137004.

51 T.-M. Chuang, M. P. Allan, J. Lee, Y. Xie, N. Ni, S. L. Bud'ko, G. S. Boebinger, P. C. Canfield and J. C. Davis, Science, 2010, 327, 181.

52 J. Kang and Z. Tešanović, Phys. Rev. B: Condens. Matter Mater. Phys., 2012, 85, 220507.

53 T. Hanaguri, K. Kitagawa, K. Matsubayashi, Y. Mazaki, Y. Uwatoko and H. Takagi, Phys. Rev. B: Condens. Matter Mater. Phys., 2012, 85, 214505.

54 M. J. Lawler, K. Fujita, J. Lee, A. R. Schmidt, Y. Kohsaka, C. K. Kim, H. Eisaki, S. Uchida, J. C. Davis, J. P. Sethna and E. A. Kim, Nature, 2010, 466, 347.

55 I. Zeljkovic, E. J. Main, T. L. Williams, M. C. Boyer, K. Chatterjee, W. D. Wise, Y. Yin, M. Zech, A. Pivonka, T. Kondo, T. Takeuchi, H. Ikuta, J. Wen, Z. Xu, G. D. Gu, E. W. Hudson and J. E. Hoffman, Nat. Mater., 2012, 11, 585.

56 M. H. Hamidian, I. a. Firmo, K. Fujita, S. Mukhopadhyay, J. W. Orenstein, H. Eisaki, S. Uchida, M. J. Lawler, E.-a. Kim and J. C. Davis, New J. Phys., 2012, 14, 053017.

57 J. H. Tapp, Z. Tang, B. Lv, K. Sasmal, B. Lorenz, P. C. W. Chu and A. M. Guloy, Phys. Rev. B: Condens. Matter Mater. Phys., 2008, 78, 060505.

58 M. F. Crommie, C. P. Lutz and D. M. Eigler, Nature, 1993, 363, 524.

59 J. E. Hoffman, K. McElroy, D.-H. Lee, K. M. Lang, H. Eisaki, S. Uchida and J. C. Davis, Science, 2002, 297, 1148.

60 Q.-H. Wang and D.-H. Lee, Phys. Rev. B: Condens. Matter Mater. Phys., 2003, 67, 020511.

61 K. McElroy, R. W. Simmonds, J. E. Hoffman, D.-H. Lee, J. Orenstein, H. Eisaki, S. Uchida and J. C. Davis, Nature, 2003, 422, 592.

62 J. E. Hoffman, A Search for Alternative Electronic Order in the High Temperature Superconductor BSCCO by Scanning Tunneling Microscopy, PhD thesis, School University of California, Berkeley, 2003. 
63 K. McElroy, G.-H. Gweon, S. Y. Zhou, J. Graf, S. Uchida, H. Eisaki, H. Takagi, T. Sasagawa, D.-H. Lee and A. Lanzara, Phys. Rev. Lett., 2006, 96, 067005.

64 U. Chatterjee, M. Shi, A. Kaminski, A. Kanigel, H. M. Fretwell, K. Terashima, T. Takahashi, S. Rosenkranz, Z. Z. Li, H. Raffy, A. Santander-Syro, K. Kadowaki, M. R. Norman, M. Randeria and J. C. Campuzano, Phys. Rev. Lett., 2006, 96, 107006.

65 M. Hashimoto, R.-H. He, J. P. Testaud, W. Meevasana, R. G. Moore, D. H. Lu, Y. Yoshida, H. Eisaki, T. P. Devereaux, Z. Hussain and Z.-X. Shen, Phys. Rev. Lett., 2011, 106, 167003.

66 Y. Kohsaka, C. Taylor, P. Wahl, A. R. Schmidt, J. Lee, K. Fujita, J. W. Alldredge, K. McElroy, J. Lee, H. Eisaki, S. Uchida, D.-H. Lee and J. C. Davis, Nature, 2008, 454, 1072.

67 W. D. Wise, K. Chatterjee, M. C. Boyer, T. Kondo, T. Takeuchi, H. Ikuta, Z. Xu, J. S. Wen, G. D. Gu, Y. Wang and E. W. Hudson, Nat. Phys., 2009, 5, 213.

68 T. Hanaguri, Y. Kohsaka, M. Ono, M. Maltseva, P. Coleman, I. Yamada, M. Azuma, M. Takano, K. Ohishi and H. Takagi, Science, 2009, 323, 923.

69 Y. He, Y. Yin, M. Zech, A. Soumyanarayanan, I. Zeljkovic, M. M. Yee, M. C. Boyer, K. Chatterjee, W. D. Wise, T. Kondo, T. Takeuchi, H. Ikuta, P. Mistark, R. S. Markiewicz, A. Bansil, S. Sachdev, E. W. Hudson and J. E. Hoffman, arXiv:1305.2778.

70 K. McElroy, J. Lee, J. A. Slezak, D.-H. Lee, H. Eisaki, S. Uchida and J. C. Davis, Science, 2005, 309, 1048.

71 I. Zeljkovic, Z. Xu, J. Wen, G. Gu, R. S. Markiewicz and J. E. Hoffman, Science, 2012, 337, 320.

72 T. Hanaguri, S. Niitaka, K. Kuroki and H. Takagi, Science, 2010, 328, 474.

73 I. I. Mazin and D. J. Singh, arXiv:1007.0255.

74 T. Hanaguri, S. Niitaka, K. Kuroki and H. Takagi, arXiv:1007.0307.

75 M. P. Allan, A. W. Rost, A. P. Mackenzie, Y. Xie, J. C. Davis, K. Kihou, C. H. Lee, A. Iyo, H. Eisaki and T.-M. Chuang, Science, 2012, 336, 563.

76 M. P. Allan, T.-M. Chuang, F. Massee, Y. Xie, N. Ni, S. L. Bud'ko, G. S. Boebinger, Q. Wang, D. S. Dessau, P. C. Canfield, M. S. Golden and J. C. Davis, Nat. Phys., 2013, 9, 220.

77 A. R. Schmidt, M. H. Hamidian, P. Wahl, F. Meier, a. V. Balatsky, J. D. Garrett, T. J. Williams, G. M. Luke and J. C. Davis, Nature, 2010, 465, 570.

78 P. Aynajian, E. H. da Silva Neto, C. V. Parker, Y. Huang, A. Pasupathy, J. Mydosh and A. Yazdani, Proc. Natl. Acad. Sci. U. S. A., 2010, 107, 10383.

79 M. H. Hamidian, A. R. Schmidt, I. A. Firmo, M. P. Allan, P. Bradley, J. D. Garrett, T. J. Williams, G. M. Luke, Y. Dubi, a. V. Balatsky and J. C. Davis, Proc. Natl. Acad. Sci. U. S. A., 2011, 108, 18233.

80 P. Aynajian, E. H. da Silva Neto, A. Gyenis, R. E. Baumbach, J. D. Thompson, Z. Fisk, E. D. Bauer and A. Yazdani, Nature, 2012, 486, 201.

81 M. P. Allan, F. Massee, D. K. Morr, J. van Dyke, A. W. Rost, A. P. Mackenzie, C. Petrovic and J. C. Davis, arXiv:1303.4416.
82 M. Maple, J. Chen, Y. Dalichaouch, T. Kohara, C. Rossel, M. Torikachvili, M. McElfresh and J. Thompson, Phys. Rev. Lett., 1986, 56, 185.

83 C. H. Booth, E. D. Bauer, A. D. Bianchi, F. Ronning, J. D. Thompson, J. L. Sarrao, J. Y. Cho, J. Y. Chan, C. Capan and Z. Fisk, Phys. Rev. B: Condens. Matter Mater. Phys., 2009, 79, 144519.

84 A. D. Bianchi, M. Kenzelmann, L. Debeer-Schmitt, J. S. White, E. M. Forgan, J. Mesot, M. Zolliker, J. Kohlbrecher, R. Movshovich, E. D. Bauer, J. L. Sarrao, Z. Fisk, C. Petrovic and M. R. Eskildsen, Science, 2008, 319, 177.

85 V. J. Emery, S. A. Kivelson and O. Zachar, Phys. Rev. B: Condens. Matter Mater. Phys., 1997, 56, 6120.

86 I. Martin and A. V. Balatsky, Phys. C, 2001, 357-360, 46.

87 Q.-H. Wang, J. Han and D.-H. Lee, Phys. Rev. B: Condens. Matter Mater. Phys., 2001, 65, 054501.

88 Z. Wang, J. Engelbrecht, S. Wang, H. Ding and S. Pan, Phys. Rev. B: Condens. Matter Mater. Phys., 2002, 65, 064509.

89 T. Cren, D. Roditchev, W. Sacks, J. Klein, J. Moussy, C. Deville-Cavellin and M. Lagues, Phys. Rev. Lett., 2000, 84, 147.

90 C. Howald, P. Fournier and A. Kapitulnik, Phys. Rev. B: Condens. Matter Mater. Phys., 2001, 64, 100504.

91 S.-H. Pan, J. P. O’Neal, R. L. Badzey, C. Chamon, H. Ding, J. R. Engelbrecht, Z. Wang, H. Eisaki, S. Uchida, A. K. Gupta, K.-W. Ng, E. W. Hudson, K. M. Lang and J. C. Davis, Nature, 2001, 413, 282.

92 K. M. Lang, V. Madhavan, J. E. Hoffman, E. W. Hudson, H. Eisaki, S. Uchida and J. C. Davis, Nature, 2002, 415, 412.

93 C. Howald, H. Eisaki, N. Kaneko and A. Kapitulnik, Proc. Natl. Acad. Sci. U. S. A., 2003, 100, 9705.

94 K. McElroy, D.-H. Lee, J. Hoffman, K. Lang, J. Lee, E. Hudson, H. Eisaki, S. Uchida and J. Davis, Phys. Rev. Lett., 2005, 94, 197005.

95 K. K. Gomes, A. N. Pasupathy, A. Pushp, S. Ono, Y. Ando and A. Yazdani, Nature, 2007, 447, 569.

96 M. C. Boyer, W. D. Wise, K. Chatterjee, M. Yi, T. Kondo, T. Takeuchi, H. Ikuta and E. W. Hudson, Nat. Phys., 2007, 3, 802.

97 A. Pushp, C. V. Parker, A. N. Pasupathy, K. K. Gomes, S. Ono, J. S. Wen, Z. Xu, G. Gu and A. Yazdani, Science, 2009, 324, 1689.

98 J. E. Hoffman, E. W. Hudson, K. M. Lang, V. Madhavan, H. Eisaki, S. Uchida and J. C. Davis, Science, 2002, 295, 466.

99 M. Vershinin, S. Misra, S. Ono, Y. Abe, Y. Ando and A. Yazdani, Science, 2004, 303, 1995.

100 C. V. Parker, P. Aynajian, E. H. da Silva Neto, A. Pushp, S. Ono, J. Wen, Z. Xu, G. Gu and A. Yazdani, Nature, 2010, 468, 677.

101 S. Zhou, H. Ding and Z. Wang, Phys. Rev. Lett., 2007, 98, 076401.

102 G. Kinoda, H. Mashima, K. Shimizu, J. Shimoyama, K. Kishio and T. Hasegawa, Phys. Rev. B: Condens. Matter Mater. Phys., 2005, 71, 020502.

103 G. Kinoda and T. Hasegawa, Phys. Rev. B: Condens. Matter Mater. Phys., 2003, 67, 224509. 
104 W. D. Wise, M. C. Boyer, K. Chatterjee, T. Kondo, T. Takeuchi, H. Ikuta, Y. Wang and E. W. Hudson, Nat. Phys., 2008, 4, 696.

105 H. Eisaki, N. Kaneko, D. Feng, A. Damascelli, P. Mang, K. Shen, Z.-X. Shen and M. Greven, Phys. Rev. B: Condens. Matter Mater. Phys., 2004, 69, 064512.

106 I. Chong, Z. Hiroi, M. Izumi, J. Shimoyama, Y. Nakayama, K. Kishio, T. Terashima, Y. Bando and M. Takano, Science, 1997, 276, 770.

107 H. Mashima, N. Fukuo, Y. Matsumoto, T. Kondo, H. Ikuta, T. Hitosugi and T. Hasegawa, Phys. Rev. B: Condens. Matter Mater. Phys., 2006, 73, 060502.

108 Y. Kohsaka, K. Iwaya, S. Satow, T. Hanaguri, M. Azuma, M. Takano and H. Takagi, Phys. Rev. Lett., 2004, 93, 097004.

109 T. Hanaguri, C. Lupien, Y. Kohsaka, D.-H. Lee, M. Azuma, M. Takano, H. Takagi and J. C. Davis, Nature, 2004, 430, 1001.

110 C. Ye, P. Cai, R. Yu, X. Zhou, W. Ruan, Q. Liu, C. Jin and Y. Wang, Nat. Commun., 2013, 4, 1365.

111 X. Leng, J. Garcia-Barriocanal, S. Bose, Y. Lee and A. Goldman, Phys. Rev. Lett., 2011, 107, 027001.

112 X. Leng, J. Garcia-Barriocanal, B. Yang, Y. Lee, J. Kinney and A. Goldman, Phys. Rev. Lett., 2012, 108, 067004.

113 A. T. Bollinger, G. Dubuis, J. Yoon, D. Pavuna, J. Misewich and I. Bozovic, Nature, 2011, 472, 458.

114 J. Garcia-Barriocanal, A. Kobrinskii, X. Leng, J. Kinney, B. Yang, S. Snyder and A. M. Goldman, Phys. Rev. B: Condens. Matter Mater. Phys., 2013, 87, 024509.

115 H. L. Edwards, J. T. Markert and A. de Lozanne, J. Vac. Sci. Technol., B, 1994, 12, 1886.

116 S. H. Pan, E. W. Hudson and J. C. Davis, Rev. Sci. Instrum., 1999, 70, 1459.

117 M. Maki, T. Nishizaki, K. Shibata and N. Kobayashi, J. Phys. Soc. Jpn., 2001, 70, 1877.

118 T. Nishizaki, K. Shibata, M. Maki and N. Kobayashi, J. Low Temp. Phys., 2003, 131, 931.

119 H. Edwards, J. Markert and A. de Lozanne, Phys. Rev. Lett., 1992, 69, 2967.

120 H. Edwards, A. Barr, J. Markert and A. de Lozanne, Phys. Rev. Lett., 1994, 73, 1154.

121 M. Maki, T. Nishizaki, K. Shibata and N. Kobayashi, Phys. Rev. B: Condens. Matter Mater. Phys., 2002, 65, 140511.

122 O. Fischer, M. Kugler, I. Maggio-Aprile, C. Berthod and C. Renner, Rev. Mod. Phys., 2007, 79, 353.

123 H. Edwards, D. Derro, A. Barr, J. Markert and A. de Lozanne, Phys. Rev. Lett., 1995, 75, 1387.

124 D. J. Derro, E. W. Hudson, K. M. Lang, S. H. Pan, J. C. Davis, J. T. Markert and A. L. de Lozanne, Phys. Rev. Lett., 2002, 88, 097002.

125 D. Morr and A. Balatsky, Phys. Rev. Lett., 2001, 87, 247002. 126 V. Zabolotnyy, S. Borisenko, A. Kordyuk, J. Geck, D. Inosov, A. Koitzsch, J. Fink, M. Knupfer, B. Büchner, S.-L. Drechsler, H. Berger, A. Erb, M. Lambacher, L. Patthey, V. Hinkov and B. Keimer, Phys. Rev. B: Condens. Matter Mater. Phys., 2007, 76, 1.
127 M. A. Hossain, J. D. F. Mottershead, D. Fournier, A. Bostwick, J. L. McChesney, E. Rotenberg, R. Liang, W. N. Hardy, G. A. Sawatzky, I. S. Elfimov, D. A. Bonn and A. Damascelli, Nat. Phys., 2008, 4, 527.

128 T. S. Nunner, B. M. Andersen, A. Melikyan and P. J. Hirschfeld, Phys. Rev. Lett., 2005, 95, 177003.

129 A. Schilling, M. Cantoni, J. D. Guo and H. R. Ott, Nature, 1993, 363, 56.

130 J. Karpinski, E. Kaldis, E. Jilek, S. Rusiecki and B. Bucher, Nature, 1988, 336, 660.

131 X. Wang, Q. Liu, Y. Lv, W. Gao, L. Yang, R. Yu, F. Li and C. Jin, Solid State Commun., 2008, 148, 538.

132 B. vom Hedt, W. Lisseck, K. Westerholt and H. Bach, Phys. Rev. B: Condens. Matter Mater. Phys., 1994, 49, 9898.

133 Y. Nakajima, T. Taen and T. Tamegai, J. Phys. Soc. Jpn., 2009, 78, 023702.

134 B. Lv, L. Deng, M. Gooch, F. Wei, Y. Sun, J. K. Meen, Y.-Y. Xue, B. Lorenz and C.-W. Chu, Proc. Natl. Acad. Sci. U. S. A., 2011, 108, 15705.

135 S. Saha, N. Butch, T. Drye, J. Magill, S. Ziemak, K. Kirshenbaum, P. Zavalij, J. Lynn and J. Paglione, Phys. Rev. B: Condens. Matter Mater. Phys., 2012, 85, 024525.

136 I. Zeljkovic, D. Huang, C.-L. Song, B. Lv, C.-W. Chu and J. E. Hoffman, Phys. Rev. B: Condens. Matter Mater. Phys., 2013, 87, 201108.

137 A. Gozar, G. Logvenov, L. F. Kourkoutis, A. T. Bollinger, L. A. Giannuzzi, D. A. Muller and I. Bozovic, Nature, 2008, 455, 782.

138 L. Goren and E. Altman, Phys. Rev. B: Condens. Matter Mater. Phys., 2011, 84, 094508.

139 R. Scanlan, A. Malozemoff and D. Larbalestier, Proc. IEEE, 2004, 92, 1639.

140 V. Selvamanickam, Y. Chen, I. Kesgin, A. Guevara, T. Shi, Y. Yao, Y. Qiao, Y. Zhang, G. Majkic, G. Carota, A. Rar, Y. Xie, J. Dackow, B. Maiorov, L. Civale, V. Braccini, J. Jaroszynski, A. Xu, D. Larbalestier and R. Bhattacharya, IEEE Trans. Appl. Supercond., 2011, 21, 3049.

141 I. Maggio-Aprile, C. Renner, A. Erb, E. Walker and O. Fischer, Phys. Rev. Lett., 1995, 75, 2754.

142 I. Maggio-Aprile, C. Renner, A. Erb, E. Walker and O. Fischer, Nature, 1997, 390, 487.

143 M. Daeumling, J. M. Seuntjens and D. C. Larbalestier, Nature, 1990, 346, 332.

144 O. M. Auslaender, L. Luan, E. W. J. Straver, J. E. Hoffman, N. C. Koshnick, E. Zeldov, D. A. Bonn, R. Liang, W. N. Hardy and K. A. Moler, Nat. Phys., 2008, 5, 35.

145 D. T. Verebelyi, D. K. Christen, R. Feenstra, C. Cantoni, A. Goyal, D. F. Lee, M. Paranthaman, P. N. Arendt, R. F. DePaula, J. R. Groves and C. Prouteau, Appl. Phys. Lett., 2000, 76, 1755.

146 J. E. Hoffman, Rep. Prog. Phys., 2011, 74, 124513.

147 J.-L. Zhang, L. Jiao, Y. Chen and H.-Q. Yuan, Front. Phys., 2012, 6, 463.

148 Y. Yin, M. Zech, T. L. Williams and J. E. Hoffman, Phys. C, 2009, 469, 535. 
149 Y. Yin, M. Zech, T. L. Williams, X. F. Wang, G. Wu, X. H. Chen and J. E. Hoffman, Phys. Rev. Lett., 2009, 102, 097002.

150 S.-H. Pan, E. W. Hudson, A. K. Gupta, K.-W. Ng, H. Eisaki, S. Uchida and J. C. Davis, Phys. Rev. Lett., 2000, 85, 1536.

151 L. Shan, Y.-L. Wang, B. Shen, B. Zeng, Y. Huang, A. Li, D. Wang, H. Yang, C. Ren, Q.-H. Wang, S. H. Pan and H.-H. Wen, Nat. Phys., 2011, 7, 325.
152 C.-L. Song, Y. Yin, M. Zech, T. Williams, M. Yee, G.-F. Chen, J.-L. Luo, N.-l. Wang, E. W. Hudson and J. E. Hoffman, arXiv:1212.3240.

153 X. He, G. Li, J. Zhang, A. Karki, R. Jin, B. Sales, A. Sefat, M. McGuire, D. Mandrus and E. Plummer, Phys. Rev. B: Condens. Matter Mater. Phys., 2011, 83, 220502.

154 B. A. Baumert, J. Supercond., 1995, 8, 175.

155 R. Wiesendanger, Rev. Mod. Phys., 2009, 81, 1495. 